TRANSACTIONS OF THE

AMERICAN MATHEMATICAL SOCIETY

Volume 362, Number 5, May 2010, Pages 2393-2414

S 0002-9947(09)05020-X

Article electronically published on December 8, 2009

\title{
MANN PAIRS
}

\author{
LOU VAN DEN DRIES AND AYHAN GÜNAYDIN
}

\begin{abstract}
Mann proved in the 1960s that for any $n \geq 1$ there is a finite set $E$ of $n$-tuples $\left(\eta_{1}, \ldots, \eta_{n}\right)$ of complex roots of unity with the following property: if $a_{1}, \ldots, a_{n}$ are any rational numbers and $\zeta_{1}, \ldots, \zeta_{n}$ are any complex roots of unity such that $\sum_{i=1}^{n} a_{i} \zeta_{i}=1$ and $\sum_{i \in I} a_{i} \zeta_{i} \neq 0$ for all nonempty $I \subseteq\{1, \ldots, n\}$, then $\left(\zeta_{1}, \ldots, \zeta_{n}\right) \in E$. Taking an arbitrary field $\mathbf{k}$ instead of $\mathbb{Q}$ and any multiplicative group in an extension field of $\mathbf{k}$ instead of the group of roots of unity, this property defines what we call a Mann pair $(\mathbf{k}, \Gamma)$. We show that Mann pairs are robust in certain ways, construct various kinds of Mann pairs, and characterize them model-theoretically.
\end{abstract}

\section{INTRODUCTION}

Throughout this paper we use the following notation and conventions. We let $\Omega$ be an ambient algebraically closed field (taken to be $\mathbb{C}$ if suggested by the context), and let $\Gamma$ be a subgroup of the multiplicative group $\Omega^{\times}$. We say that $\Gamma$ has finite rank if $\Gamma$ has a finitely generated subgroup $\Gamma_{0}$ such that $\Gamma / \Gamma_{0}$ is a torsion group. We let $m, n$ range over $\mathbb{N}=\{0,1,2, \ldots\}$. For $r \in \Omega$ and $\vec{s}=\left(s_{1}, \ldots, s_{n}\right) \in \Omega^{n}$ we put $r \vec{s}:=\left(r s_{1}, \ldots, r s_{n}\right) \in \Omega^{n}$. In particular, if $\alpha \in \Gamma$ and $\vec{\gamma} \in \Gamma^{n}$, then $\alpha \vec{\gamma} \in \Gamma^{n}$. We let $\mathbb{U} \subseteq \mathbb{C}^{\times}$be the group of complex roots of unity, and $\mathbb{Q}^{\text {ac }} \subseteq \mathbb{C}$ the field of complex algebraic numbers.

Let $a_{1}, \ldots, a_{n} \in \Omega, n \geq 1$, and consider the equation

$$
a_{1} x_{1}+\cdots+a_{n} x_{n}=1 .
$$

A solution of $(*)$ is a tuple $\left(s_{1}, \ldots, s_{n}\right) \in \Omega^{n}$ such that $a_{1} s_{1}+\cdots+a_{n} s_{n}=1$; such a solution is said to be nondegenerate if $\sum_{i \in I} a_{i} s_{i} \neq 0$ for all nonempty $I \subseteq\{1, \ldots, n\}$, and is said to be in $\Gamma$ if $\left(s_{1}, \ldots, s_{n}\right) \in \Gamma^{n}$.

In [5] we defined $\Gamma$ to have the Mann property if for all $n \geq 1$ and nonzero $a_{1}, \ldots, a_{n}$ in the prime field of $\Omega$ the equation $(*)$ has only finitely many nondegenerate solutions in $\Gamma$; equivalently, for all $n \geq 1$ and $a_{1}, \ldots, a_{n} \in \Omega^{\times}$the equation $(*)$ has only finitely many non-degenerate solutions in $\Gamma$, see [5]. The Mann property is a Mordell-Lang type property. Mann [12] gave an effective proof that $\mathbb{U}$ has the Mann property. More generally, but less effectively, 6], 11, and 14. show that if $\Omega$ has characteristic zero and $\Gamma$ has finite rank, then $\Gamma$ has the Mann property. In [5] we studied the model theory of structures $(\Omega, \Gamma)$ when $\Gamma$ has the Mann property. We now define a uniform version of the Mann property involving also a subfield of $\Omega$. From now on $\mathbf{k}$ is a subfield of $\Omega$.

Received by the editors November 1, 2007.

2010 Mathematics Subject Classification. Primary 03C35, 03C60, 03C98, 11 U09.

(C) 2009 American Mathematical Society

Reverts to public domain 28 years from publication 
Call $(\mathbf{k}, \Gamma)$ a Mann pair if for each $n \geq 1$ there is a finite $E \subseteq \Gamma^{n}$ such that for all $a_{1}, \ldots, a_{n} \in \mathbf{k}^{\times}$, all nondegenerate solutions of $(*)$ in $\Gamma$ lie in $E$.

Suppose $(\mathbf{k}, \Gamma)$ is a Mann pair. It is obvious that then $\Gamma$ has the Mann property and that if $\mathbf{k}^{\prime}$ is a subfield of $\mathbf{k}$ and $\Gamma^{\prime}$ a subgroup of $\Gamma$, then $\left(\mathbf{k}^{\prime}, \Gamma^{\prime}\right)$ is also a Mann pair. Taking $n=1$ in the definition we see that $\mathbf{k}^{\times} \cap \Gamma$ is finite, and so all elements of $\mathbf{k}^{\times} \cap \Gamma$ are roots of unity.

Actually, Theorem 1 of $[12$ implies that $(\mathbb{Q}, \mathbb{U})$ is a Mann pair; conversely, Corollary 5.1 below says that if $(\mathbb{Q}, \Gamma)$ is a Mann pair and $\Gamma \subseteq\left(\mathbb{Q}^{\text {ac }}\right)^{\times}$, then $\Gamma \subseteq \mathbb{U}$. Thus $\left(\mathbb{Q},(1+\sqrt{2})^{\mathbb{Z}}\right)$ is not a Mann pair, although $(1+\sqrt{2})^{\mathbb{Z}} \subseteq\left(\mathbb{Q}^{\text {ac }}\right)^{\times}$has the Mann property and $\mathbb{Q}^{\times} \cap(1+\sqrt{2})^{\mathbb{Z}}=\{1\}$. In Section 2 we indicate simple examples of Mann pairs, such as:

(1) $\left(\mathbb{Q}^{\text {ac }}, \exp \left(\mathbb{Q}^{\text {ac }}\right)\right)$ is a Mann pair (Lindemann's theorem).

(2) If $v: K^{\times} \rightarrow v\left(K^{\times}\right)$is a valuation on a subfield $K$ of $\Omega$ and $\mathbf{k}$ is a subfield of $K$ and $\Gamma$ a subgroup of $K^{\times}$such that $v$ is trivial on $\mathbf{k}$ and injective on $\Gamma$, then $(\mathbf{k}, \Gamma)$ is a Mann pair.

A more substantial source of Mann pairs is the following:

Theorem 1.1. Suppose $\Omega$ has characteristic zero, $\mathbf{k}$ is algebraically closed, $\mathbf{k}^{\times} \cap \Gamma=$ $\{1\}$, and $\Gamma$ has finite rank. Then $(\mathbf{k}, \Gamma)$ is a Mann pair.

This is an analogue for Mann pairs of the result from [6, [1], 14 that was mentioned earlier. In Section 3 we first deal with the special case that $\Gamma$ is finitely generated and $\mathbf{k}(\Gamma)$ has transcendence degree 1 over $\mathbf{k}$; the key here is a height bound by Brownawell and Masser [3]. With this special case as a stepping stone and using various facts established in Sections 4 and 5 we prove Theorem 1.1 in Section 6. This proof is effective, at least in principle, but we do not elaborate upon this aspect in the present paper. Section 5 also describes some curious Mann pairs in positive characteristic as well as some Mann pairs $\left(\mathbb{Q}_{p}, \Gamma\right)$ obtained by Teichmüller lifting.

Next we consider the structure $(\Omega, \mathbf{k}, \Gamma)$, that is, the algebraically closed field $\Omega$ with distinguished subsets $\mathbf{k}$ and $\Gamma$. In Sections 4 and 7 we show:

Theorem 1.2. The following are equivalent:

(1) $(\mathbf{k}, \Gamma)$ is a Mann pair;

(2) for each $n \geq 1$ the set $\left\{(x, y) \in \mathbf{k}^{n} \times \Gamma^{n}: x_{1} y_{1}+\cdots+x_{n} y_{n}=0\right\}$ is a finite union of sets $X \times Y$ where $X \subseteq \mathbf{k}^{n}$ is definable in the field $\mathbf{k}$ and $Y \subseteq \Gamma^{n}$ is definable in the group $\Gamma$;

(3) for all $m, n$, every subset of $\mathbf{k}^{m} \times \Gamma^{n}$ definable in $(\Omega, \mathbf{k}, \Gamma)$ is a finite union of sets $X \times Y$ with $X \subseteq \mathbf{k}^{m}$ definable in the field $\mathbf{k}$ and $Y \subseteq \Gamma^{n}$ definable in the group $\Gamma$.

Here and below, "definable" means "definable with parameters" unless we specify otherwise. Thus (3) says that the structure induced by $(\Omega, \mathbf{k}, \Gamma)$ on $\mathbf{k}$ and $\Gamma$ (using parameters from $\Omega$ ) is just the field structure of $\mathbf{k}$ and the group structure of $\Gamma$, respectively, and that these two structures only interact trivially inside $(\Omega, \mathbf{k}, \Gamma)$.

In Section 8 we prove that for Mann pairs $(\mathbf{k}, \Gamma)$ with $[\Omega: \mathbf{k}]>2$, the complete theory $\operatorname{Th}(\Omega, \mathbf{k}, \Gamma)$ is determined by $\operatorname{Th}(\mathbf{k})$ and $\operatorname{Th}(\Gamma)$ after adding names for enough elements of $\mathbf{k}$ and $\Gamma$ to witness that $(\mathbf{k}, \Gamma)$ is a Mann pair. Under the additional assumption that $\mathbf{k}$ is algebraically closed we show that $(\Omega, \mathbf{k}, \Gamma)$ is stable, and we make further observations along these lines.

We let $\mathbf{k}^{\text {ac }}$ be the algebraic closure in $\Omega$ of any subfield $\mathbf{k}$ of $\Omega$. 


\section{Some EXAmples of MANn pairs}

It will be useful to also consider homogeneous linear equations. Let $n \geq 1$ and let $a_{1}, \ldots, a_{n} \in \Omega$. A solution of the homogeneous linear equation

$$
a_{1} x_{1}+\cdots+a_{n} x_{n}=0
$$

is a tuple $\left(s_{1}, \ldots, s_{n}\right) \in \Omega^{n}$ such that $a_{1} s_{1}+\cdots+a_{n} s_{n}=0$; such a solution is said to be nondegenerate if $\sum_{i \in I} a_{i} s_{i} \neq 0$ for all nonempty proper subsets $I$ of $\{1, \ldots, n\}$, and is said to be in $\Gamma$ if $\left(s_{1}, \ldots, s_{n}\right) \in \Gamma^{n}$.

2.1. Alternative definition of Mann pairs. The equivalence of (1) and (2) in the next lemma expresses being a Mann pair in terms of homogeneous linear equations. Assuming $\mathbf{k}^{\times} \cap \Gamma$ is finite, the equivalence between (1) and (3) expresses $(\mathbf{k}, \Gamma)$ being a Mann pair purely in terms of the subgroup $G=\mathbf{k}^{\times} \Gamma$ of $\Omega^{\times}$generated by $\mathbf{k}^{\times}$and $\Gamma$.

Lemma 2.1. Let $G=\mathbf{k}^{\times} \Gamma$. Then the following are equivalent:

(1) $(\mathbf{k}, \Gamma)$ is a Mann pair;

(2) for each $n \geq 1$ there is a finite $\Gamma(n) \subseteq \Gamma^{n}$ such that each nondegenerate solution in $\Gamma$ of each equation $a_{1} x_{1}+\cdots+a_{n} x_{n}=0$ with $a_{1}, \ldots, a_{n} \in \mathbf{k}^{\times}$ lies in $\alpha \Gamma(n)$ for some $\alpha \in \Gamma$;

(3) $\mathbf{k}^{\times} \cap \Gamma$ is finite, and for each $n \geq 1$ there is a finite $G(n) \subseteq G^{n}$ such that each nondegenerate solution in $G$ of the equation

$$
\begin{gathered}
x_{1}+\cdots+x_{n}=1 \\
\text { equals }\left(c_{1} g_{1}, \ldots, c_{n} g_{n}\right) \text { with } c_{1}, \ldots, c_{n} \in \mathbf{k}^{\times} \text {and }\left(g_{1}, \ldots, g_{n}\right) \in G(n) .
\end{gathered}
$$

Proof. Let $n \geq 1$. First note that if $a_{1}, \ldots, a_{n} \in \mathbf{k}^{\times}$and $\left(s_{1}, \ldots, s_{n}\right) \in \Omega^{n}$, then $\left(s_{1}, \ldots, s_{n}\right)$ is a nondegenerate solution of $a_{1} x_{1}+\cdots+a_{n} x_{n}=1$ iff $\left(s_{1}, \ldots, s_{n}, 1\right)$ is a nondegenerate solution of $a_{1} x_{1}+\cdots+a_{n} x_{n}-x_{n+1}=0$.

Therefore, if (1) holds, witnessed by $E$ for our given $n$ as in the definition of Mann pair in the Introduction, then (2) holds with

$$
\Gamma(n+1):=\left\{\left(\gamma_{1}, \ldots, \gamma_{n}, 1\right):\left(\gamma_{1}, \ldots, \gamma_{n}\right) \in E\right\} .
$$

Conversely, if (2) holds, take $\Gamma(n+1)$ such that $\gamma_{n+1}=1$ for every $\left(\gamma_{1}, \ldots, \gamma_{n}, \gamma_{n+1}\right)$ from $\Gamma(n+1)$, and then (1) holds with

$$
E:=\left\{\left(\gamma_{1}, \ldots, \gamma_{n}\right):\left(\gamma_{1}, \ldots, \gamma_{n}, 1\right) \in \Gamma(n+1)\right\} .
$$

Next, observe that if (1) holds, witnessed by $E$ for our given $n$, then (3) holds with $G(n):=E$. Conversely, if (3) holds, then in (3) we can take $G(n) \subseteq \Gamma^{n}$, and then (1) holds with

$$
E:=\left\{\left(\alpha_{1} \gamma_{1}, \ldots, \alpha_{n} \gamma_{n}\right) \in \Gamma^{n}: \alpha_{1}, \ldots, \alpha_{n} \in \mathbf{k}^{\times} \cap \Gamma,\left(\gamma_{1}, \ldots, \gamma_{n}\right) \in G(n)\right\} .
$$

Corollary 2.2. Suppose $(\mathbf{k}, \Gamma)$ is a Mann pair, and $\Gamma^{\prime}$ is a subgroup of $\mathbf{k}^{\times} \Gamma$ such that $\mathbf{k}^{\times} \cap \Gamma^{\prime}$ is finite. Then $\left(\mathbf{k}, \Gamma^{\prime}\right)$ is a Mann pair. 
2.2. Easy Mann pairs. These are provided by the next lemma.

Lemma 2.3. Suppose $\Gamma$ is torsion-free. Then the following are equivalent:

(1) for all $n \geq 1$ and $a_{1}, \ldots, a_{n} \in \mathbf{k}^{\times}$, the equation $a_{1} x_{1}+\cdots+a_{n} x_{n}=1$ has no nondegenerate solution in $\Gamma$ that is different from $(1, \ldots, 1)$;

(2) whenever $n \geq 1$ and $\gamma_{1}, \ldots, \gamma_{n} \in \Gamma$ are multiplicatively independent, then they are algebraically independent over $\mathbf{k}$.

If these conditions are satisfied, then $(\mathbf{k}, \Gamma)$ is a Mann pair.

Proof. Apply Lemma 8.2 in [5] (but $\Gamma, E, G$ in that lemma are $\{1\}, \mathbf{k}, \Gamma$ in the present lemma).

Since $\pi \notin \mathbb{Q}^{\text {ac }}$, the group $\exp \left(\mathbb{Q}^{\text {ac }}\right) \subseteq \mathbb{C}^{\times}$is torsion-free, and by Lindemann's theorem on exponentials ([9], Appendix 1), condition (2) of the lemma is satisfied with $\mathbf{k}=\mathbb{Q}^{\text {ac }} \subseteq \mathbb{C}$ and $\Gamma=\exp \left(\mathbb{Q}^{\mathrm{ac}}\right)$. Thus $\left(\mathbb{Q}^{\mathrm{ac}}, \exp \left(\mathbb{Q}^{\mathrm{ac}}\right)\right)$ is a Mann pair. Here is another application of the lemma, which applies for example to Hahn fields $K=\mathbf{k}((\Gamma))$.

Corollary 2.4. Let $v: K^{\times} \rightarrow v\left(K^{\times}\right)$be a valuation on a subfield $K$ of $\Omega$. Suppose $\mathbf{k}$ is a subfield of $K$ and $\Gamma$ a subgroup of $K^{\times}$such that $v$ is trivial on $\mathbf{k}$ and injective on $\Gamma$. Then condition (2) of Lemma 2.3 is satisfied, and so $(\mathbf{k}, \Gamma)$ is a Mann pair.

A group with the Mann property can produce a Mann pair in an elementary extension as follows:

Corollary 2.5. Suppose $G$ is a subgroup of $\mathbf{k}^{\times}$with the Mann property, $\left(\mathbf{k}^{*}, G^{*}\right)$ is an elementary extension of $(\mathbf{k}, G)$, and $\Gamma$ is a subgroup of $G^{*}$ such that $\mathbf{k}^{\times} \cap \Gamma=\{1\}$. Then $(\mathbf{k}, \Gamma)$ is a Mann pair.

Proof. Note that $\Gamma$ is torsion-free. Let $a_{1}, \ldots, a_{n} \in \mathbf{k}^{\times}, n \geq 1$. Then the equation $a_{1} x_{1}+\cdots+a_{n} x_{n}=1$ has the same finite number of nondegenerate solutions in $G$ as in $G^{*}$, so cannot have nondegenerate solutions in $\Gamma$ different from $(1, \ldots, 1)$, the unique element of $G^{n} \cap \Gamma^{n}$. Hence condition (1) of Lemma 2.3 is satisfied, and so $(\mathbf{k}, \Gamma)$ is a Mann pair.

If $\mathbf{k}$ has positive characteristic, then Lemma 2.3 is the only source of Mann pairs $(\mathbf{k}, \Gamma)$ with torsion-free $\Gamma$ :

Corollary 2.6. Suppose $\mathbf{k}$ has positive characteristic, $\Gamma$ is torsion-free, and $(\mathbf{k}, \Gamma)$ is a Mann pair. Then conditions (1) and (2) of Lemma 2.3 are satisfied.

Proof. Let $a_{1}, \ldots, a_{n} \in \mathbf{k}^{\times}, n \geq 1$, and suppose $\left(\gamma_{1}, \ldots, \gamma_{n}\right) \in \Gamma^{n}$ is a nondegenerate solution of $a_{1} x_{1}+\cdots+a_{n} x_{n}=1$. Let $\phi$ be the Frobenius map on k. Then for each $m$ we have a nondegenerate solution

$$
\left(\phi^{m}\left(\gamma_{1}\right), \ldots, \phi^{m}\left(\gamma_{n}\right)\right) \in \Gamma^{n}
$$

of the equation $\phi^{m}\left(a_{1}\right) x_{1}+\cdots+\phi^{m}\left(a_{n}\right) x_{n}=1$. By the equivalence $(1) \Leftrightarrow(2)$ of Lemma 2.1 this yields that all $\gamma_{i}$ are roots of unity, so equal to 1 by torsion-freeness of $\Gamma$. 


\section{MANN PAIRS IN FUNCTION FIELDS OF ONE VARIABLE}

Let $\mathbf{k}$ be algebraically closed, and let $F \subseteq \Omega$ be a function field of one variable over $\mathbf{k}$; that is, $F$ is a field extension of finite degree of $\mathbf{k}(t)$ for some $t \in \Omega \backslash \mathbf{k}$. In particular, $F$ has transcendence degree 1 over $\mathbf{k}$. Below we use some standard facts about such function fields; for proofs of these facts, see Chapter I, §2 of [10].

Let $\mathcal{R}(F \mid \mathbf{k})$, the Riemann space of $F$ over $\mathbf{k}$, be the set of all valuations $v$ : $F^{\times} \rightarrow \mathbb{Z}$ on $F$ with value group $v\left(F^{\times}\right)=\mathbb{Z}$ that are trivial on $\mathbf{k}$. We let $v$ range over $\mathcal{R}(F \mid \mathbf{k})$. For each $f \in F^{\times}$we have $v(f) \neq 0$ for only finitely many $v$, and $\sum_{v} v(f)=0$. Let $\mathcal{D}(F \mid \mathbf{k})$ be the group of divisors of $F$ over $\mathbf{k}$; that is,

$$
\mathcal{D}(F \mid \mathbf{k}):=\bigoplus_{v} \mathbb{Z} v
$$

is the free abelian group on the Riemann space of $F$ over $\mathbf{k}$. To $f \in F^{\times}$we assign its principal divisor $(f):=\sum_{v} v(f) v \in \mathcal{D}(F \mid \mathbf{k})$. The group morphism

$$
F^{\times} \rightarrow \mathcal{D}(F \mid \mathbf{k}), \quad f \mapsto(f)
$$

has kernel $\mathbf{k}^{\times}$. In particular, if $\Gamma$ is a subgroup of $F^{\times}$with $\mathbf{k}^{\times} \cap \Gamma=\{1\}$, then this morphism is injective on $\Gamma$, and so the image of $\Gamma$ under this morphism is an isomorphic copy of $\Gamma$ inside the free abelian group $\mathcal{D}(F \mid \mathbf{k})$. It follows that each such $\Gamma$ is free as an abelian group.

Given finite $S \subseteq \mathcal{R}(F \mid \mathbf{k})$, an $S$-unit is an element $u \in F^{\times}$such that $v(u)=0$ for all $v \notin S$.

Lemma 3.1. Let $L$ be a finite-dimensional $\mathbf{k}$-linear subspace of $F$ and let $\Gamma \subseteq F^{\times}$ be finitely generated with $\mathbf{k}^{\times} \cap \Gamma=\{1\}$. Then $L \cap \Gamma$ is finite.

Proof. Let $b_{1}, \ldots, b_{m}$ be a basis of the $\mathbf{k}$-linear space $L$, and let $\gamma_{1}, \ldots, \gamma_{n}$ generate the group $\Gamma$. Take a finite $S \subseteq \mathcal{R}(F \mid \mathbf{k})$ such that all $b_{i}$ and $\gamma_{j}$ are $S$-units. Take a natural number $d$ such that $v\left(b_{i}\right) \geq-d$ for all $v \in S$ and $i=1, \ldots, m$. Then $v(f) \geq 0$ for all $f \in L$ and $v$ outside $S$, and $v(f) \geq-d$ for all $f \in L$ and $v \in S$.

Suppose now that $\gamma \in L \cap \Gamma$. Then $v(\gamma)=0$ for $v$ outside $S$ and $v(\gamma) \geq-d$ for $v \in S$. In view of $\sum_{v} v(\gamma)=0$, this gives $v(\gamma) \leq|S| d$ for all $v \in S$. It follows that the image of $L \cap \Gamma$ in $\mathcal{D}(F \mid \mathbf{k})$ is finite, and thus $L \cap \Gamma$ is finite.

Let $u_{1}, \ldots, u_{n} \in F$ not be all zero. We define their height by

$$
H\left(u_{1}, \ldots, u_{n}\right):=-\sum_{v} \min \left\{v\left(u_{1}\right), \ldots, v\left(u_{n}\right)\right\} .
$$

This height is projective: $H\left(f u_{1}, \ldots, f u_{n}\right)=H\left(u_{1}, \ldots, u_{n}\right)$ for $f \in F^{\times}$.

Example. Let $F=\mathbf{k}(t)$ with $t$ transcendental over $\mathbf{k}$. Suppose that the polynomials $u_{1}, \ldots, u_{n} \in \mathbf{k}[t]$ have no common zero in $\mathbf{k}, n \geq 1$. It is easy to check that then $H\left(u_{1}, \ldots, u_{n}\right)=\max \left\{\operatorname{deg}_{t} u_{1}, \ldots, \operatorname{deg}_{t} u_{n}\right\}$.

The following important bound is from $[3]$ :

Let $\Omega$ have characteristic zero, let $\mathrm{g}$ be the genus of the function field $F \mid \mathbf{k}$, and let $S$ be a finite subset of $\mathcal{R}(F \mid \mathbf{k})$ and $n \geq 2$. Suppose $u_{1}, \ldots, u_{n}$ are $S$-units and $\left(u_{1}, \ldots, u_{n}\right)$ is a nondegenerate solution of $x_{1}+\cdots+x_{n}=0$. Then

$$
H\left(u_{1}, \ldots, u_{n}\right) \leq \frac{1}{2}(n-1)(n-2)\{|S|+\max (0,2 \mathrm{~g}-2)\} .
$$

In combination with the previous lemma this has the following consequence. 
Corollary 3.2. Suppose $\Omega$ has characteristic zero and $\Gamma \subseteq F^{\times}$is finitely generated with $\mathbf{k}^{\times} \cap \Gamma=\{1\}$. Then $(\mathbf{k}, \Gamma)$ is a Mann pair.

Proof. Take a finite $S \subseteq \mathcal{R}(F \mid \mathbf{k})$ such that all $\gamma \in \Gamma$ are $S$-units. Let $n \geq 2$ and let $a_{1}, \ldots, a_{n} \in \mathbf{k}^{\times}$and $\gamma_{1}, \ldots, \gamma_{n} \in \Gamma$ be such that $\left(\gamma_{1}, \ldots, \gamma_{n}\right)$ is a nondegenerate solution of $a_{1} x_{1}+\cdots+a_{n} x_{n}=0$. Dividing by $\gamma_{n}$ we arrange $\gamma_{n}=1$, and we need to show that this leaves only finitely many possibilities for $\left(\gamma_{1}, \ldots, \gamma_{n-1}\right)$. Now by the bound above we have $v\left(\gamma_{i}\right) \geq-N$ for all $v \in S$ and $i=1, \ldots, n-1$, where $N:=\frac{1}{2}(n-1)(n-2)\{|S|+\max (0,2 \mathrm{~g}-2)\}$, so each $\gamma_{i}$ lies in the $\mathbf{k}$-linear subspace

$$
L:=\{f \in F: v(f) \geq 0 \text { for all } v \notin S, v(f) \geq-N \text { for all } v \in S\}
$$

of $F$. Now $L$ is finite-dimensional by [10, p. 7. In view of Lemma 3.1 this gives the desired finiteness.

Example. To illustrate the effective nature of this proof, assume that $\Omega$ has characteristic zero, and consider the case $F=\mathbf{k}(t)$ of a rational function field ( $\mathrm{so} g=0$ ), where $\Gamma$ is generated as a group by

$$
t-c_{1}, \ldots, t-c_{M}, \quad \text { with distinct } c_{1}, \ldots, c_{M} \in \mathbf{k} .
$$

Let $n \geq 2$ be given. Call a tuple $\left(\gamma_{1}, \ldots, \gamma_{n}\right) \in \Gamma^{n}$ reduced if all $\gamma_{i}$ lie in $\mathbf{k}[t]$ and $\gamma_{1}, \ldots, \gamma_{n}$ have no common zero in $\mathbf{k}$ when viewed as polynomials in $t$ over $\mathbf{k}$. Let $a_{1}, \ldots, a_{n} \in \mathbf{k}^{\times}$. Any nondegenerate solution in $\Gamma$ of the equation

$$
a_{1} x_{1}+\cdots+a_{n} x_{n}=0
$$

can be multiplied by an element of $\Gamma$ to give a nondegenerate reduced solution $\left(\gamma_{1}, \ldots, \gamma_{n}\right)$. In this situation the inequality from 3 yields

$$
\max \left\{\operatorname{deg}_{t} \gamma_{1}, \ldots, \operatorname{deg}_{t} \gamma_{n}\right\} \leq \frac{1}{2}(n-1)(n-2)(M+1),
$$

which is satisfied by only finitely many reduced tuples $\left(\gamma_{1}, \ldots, \gamma_{n}\right)$, which we can list explicitly. Given a reduced tuple $\left(\gamma_{1}, \ldots, \gamma_{n}\right)$ satisfying the inequality, the existence of $a_{1}, \ldots, a_{n} \in \mathbf{k}^{\times}$such that $\left(\gamma_{1}, \ldots, \gamma_{n}\right)$ is a nondegenerate solution of $a_{1} x_{1}+\cdots+a_{n} x_{n}=0$ is equivalent (effectively) to the existence of a solution to a certain finite system of linear equations and inequations with coefficients in the field $\mathbb{Q}\left(c_{1}, \ldots, c_{M}\right)$.

\section{Allowing coefficients from $\Omega$}

In this section we consider $\Gamma$ as acting on itself by multiplication, making $\Gamma$ into a $\Gamma$-set as defined in [5], §4. In particular, any subset of $\Gamma^{n}$ definable in the $\Gamma$-set $\Gamma$ is definable in the group $\Gamma$. Our aim is to prove the following result and some related facts.

Proposition 4.1. Let $(\mathbf{k}, \Gamma)$ be a Mann pair and $r_{1}, \ldots, r_{n} \in \Omega$. Then

$$
\left\{(x, y) \in \mathbf{k}^{n} \times \Gamma^{n}: r_{1} x_{1} y_{1}+\cdots+r_{n} x_{n} y_{n}=0\right\}
$$

is a finite union of sets $X \times Y$ with $X$ a $\mathbf{k}$-linear subspace of $\mathbf{k}^{n}$ and $Y \subseteq \Gamma^{n}$ defined in the $\Gamma$-set $\Gamma$ by a conjunction of atoms in the language of $\Gamma$-sets.

We first show this for $r_{1}, \ldots, r_{n} \in \mathbf{k}$, next for $r_{1}, \ldots, r_{n} \in \mathbf{k}(\Gamma)$, and then in general. 
4.1. Notation. In this section we let $\vec{a}=\left(a_{1}, \ldots, a_{n}\right)$ and $\vec{b}=\left(b_{1}, \ldots, b_{n}\right)$ range over $\mathbf{k}^{n}$, and $\alpha, \beta, \gamma$ over $\Gamma$, and

$$
\vec{\alpha}=\left(\alpha_{1}, \ldots, \alpha_{n}\right), \quad \vec{\beta}=\left(\beta_{1}, \ldots, \beta_{n}\right), \quad \vec{\gamma}=\left(\gamma_{1}, \ldots, \gamma_{n}\right)
$$

over $\Gamma^{n}$. Let $n \geq 1$ and put

$$
\begin{aligned}
\Sigma_{n}(\mathbf{k}, \Gamma): & =\left\{(\vec{a}, \vec{\gamma}) \in \mathbf{k}^{n} \times \Gamma^{n}: a_{1} \gamma_{1}+\cdots+a_{n} \gamma_{n}=0\right\}, \\
\Sigma_{n}(\mathbf{k}, \Gamma ; \vec{\gamma}): & =\left\{\vec{a} \in \mathbf{k}^{n}:(\vec{a}, \vec{\gamma}) \in \Sigma_{n}(\mathbf{k}, \Gamma)\right\} .
\end{aligned}
$$

Imposing nondegeneracy yields the set $\Sigma_{n}^{\text {nd }}(\mathbf{k}, \Gamma)$ of all $(\vec{a}, \vec{\gamma}) \in\left(\mathbf{k}^{\times}\right)^{n} \times \Gamma^{n}$ such that $\vec{\gamma}$ is a nondegenerate solution of $a_{1} x_{1}+\cdots+a_{n} x_{n}=0$. We also introduce for each $\vec{\gamma}$ the corresponding section

$$
\Sigma_{n}^{\mathrm{nd}}(\mathbf{k}, \Gamma ; \vec{\gamma}):=\left\{\vec{a} \in\left(\mathbf{k}^{\times}\right)^{n}:(\vec{a}, \vec{\gamma}) \in \Sigma_{n}^{\mathrm{nd}}(\mathbf{k}, \Gamma)\right\} .
$$

If $(\vec{a}, \vec{\gamma}) \in \Sigma_{n}^{\text {nd }}(\mathbf{k}, \Gamma)$, then $(\vec{a}, \alpha \vec{\gamma}) \in \Sigma_{n}^{\text {nd }}(\mathbf{k}, \Gamma)$, so $\Sigma_{n}^{\text {nd }}(\mathbf{k}, \Gamma)$ is a union of sets of the form $\Sigma_{n}^{\text {nd }}(\mathbf{k}, \Gamma ; \vec{\gamma}) \times \Gamma \vec{\gamma}$. Being a Mann pair can now be expressed as follows:

$(\mathbf{k}, \Gamma)$ is a Mann pair iff for each $n \geq 1$ there is a finite $\Gamma(n) \subseteq \Gamma^{n}$ such that

$$
\Sigma_{n}^{\mathrm{nd}}(\mathbf{k}, \Gamma)=\bigcup_{\vec{\gamma} \in \Gamma(n)} \Sigma_{n}^{\mathrm{nd}}(\mathbf{k}, \Gamma ; \vec{\gamma}) \times \Gamma \vec{\gamma} .
$$

Whenever $(\mathbf{k}, \Gamma)$ is a Mann pair we let $\Gamma(n)$ for $n \geq 1$ be as above.

We also want to allow coefficients outside $\mathbf{k}$ and accordingly, given $\vec{r}=\left(r_{1}, \ldots, r_{n}\right)$ $\in \Omega^{n}$, we let $\Sigma(\vec{r}, \mathbf{k}, \Gamma)$ be the set of all

$$
(\vec{a}, \vec{\gamma})=\left(a_{1}, \ldots, a_{n}, \gamma_{1}, \ldots, \gamma_{n}\right) \in \mathbf{k}^{n} \times \Gamma^{n}
$$

such that $\vec{\gamma}$ is a solution of $r_{1} a_{1} x_{1}+\cdots+r_{n} a_{n} x_{n}=0$, and let $\Sigma^{\text {nd }}(\vec{r}, \mathbf{k}, \Gamma)$ be the set of all $(\vec{a}, \vec{\gamma}) \in\left(\mathbf{k}^{\times}\right)^{n} \times \Gamma^{n}$ such that $\vec{\gamma}$ is a nondegenerate solution of $r_{1} a_{1} x_{1}+$ $\cdots+r_{n} a_{n} x_{n}=0$. In particular, $\Sigma^{\text {nd }}(\vec{r}, \mathbf{k}, \Gamma)=\emptyset$ if $n \geq 2$ and $r_{i}=0$ for some $i \in\{1, \ldots, n\}$. Also, let $\Sigma^{\text {nd }}(\vec{r}, \mathbf{k}, \Gamma ; \vec{\gamma})$ be the set of $\vec{a} \in\left(\mathbf{k}^{\times}\right)^{n}$ such that $(\vec{a}, \vec{\gamma}) \in$ $\Sigma^{\text {nd }}(\vec{r}, \mathbf{k}, \Gamma)$.

4.2. Mann pairs over $F$. Besides $\mathbf{k}$ we also let $F$ denote a subfield of $\Omega$. Usually, $\mathbf{k}$ serves as the base field, and $F$ will be an extension of $\mathbf{k}$.

We say that $(\mathbf{k}, \Gamma)$ is a Mann pair over $F$ if for every tuple $\vec{r}=\left(r_{1}, \ldots, r_{n}\right)$ from $F^{\times}, n \geq 2$, there is a finite subset $\Gamma(\vec{r})$ of $\Gamma^{n}$, such that

$$
\Sigma^{\mathrm{nd}}(\vec{r}, \mathbf{k}, \Gamma)=\bigcup_{\vec{\gamma} \in \Gamma(\vec{r})} \Sigma^{\mathrm{nd}}(\vec{r}, \mathbf{k}, \Gamma ; \vec{\gamma}) \times \Gamma \vec{\gamma} .
$$

It is clear that $(\mathbf{k}, \Gamma)$ is a Mann pair iff it is a Mann pair over $\mathbf{k}$. We proceed to show that if $(\mathbf{k}, \Gamma)$ is a Mann pair, then it is a Mann pair over $\Omega$.

Let $\vec{r}=\left(r_{1}, \ldots, r_{n}\right) \in F^{n}, n \geq 1$. Let $\mathcal{P}$ be a partition of $\{1, \ldots, n\}$ into distinct sets. Then we define $\Sigma_{\mathcal{P}}(\vec{r}, \mathbf{k}, \Gamma)$ to be the set of all

$$
\left(a_{1}, \ldots, a_{n}, \beta_{1}, \ldots, \beta_{n}\right) \in \mathbf{k}^{n} \times \Gamma^{n}
$$

such that for $I \in \mathcal{P}$ the tuple $\left(\beta_{i}\right)_{i \in I}$ is a nondegenerate solution of

$$
\sum_{i \in I} r_{i} a_{i} x_{i}=0 .
$$

With $\vec{r}_{I}:=\left(r_{i}\right)_{i \in I}$ for $I \in \mathcal{P}$, this means

$$
\Sigma_{\mathcal{P}}(\vec{r}, \mathbf{k}, \Gamma)=\prod_{I \in \mathcal{P}} \Sigma^{\mathrm{nd}}\left(\vec{r}_{I}, \mathbf{k}, \Gamma\right) .
$$


Suppose now that $(\mathbf{k}, \Gamma)$ is a Mann pair over $F$. Then we have for $I \in \mathcal{P}$ a finite $\Gamma\left(\vec{r}_{I}\right) \subseteq \Gamma^{I}$ such that

$$
\Sigma^{\mathrm{nd}}\left(\vec{r}_{I}, \mathbf{k}, \Gamma\right)=\bigcup_{\vec{\gamma} \in \Gamma\left(\vec{r}_{I}\right)} \Sigma^{\mathrm{nd}}\left(\vec{r}_{I}, \mathbf{k}, \Gamma ; \vec{\gamma}\right) \times \Gamma \vec{\gamma}
$$

It follows that

$$
\Sigma_{\mathcal{P}}(\vec{r}, \mathbf{k}, \Gamma)=\prod_{I \in \mathcal{P}} \bigcup_{\vec{\gamma} \in \Gamma\left(\vec{r}_{I}\right)} \Sigma^{\mathrm{nd}}\left(\vec{r}_{I}, \mathbf{k}, \Gamma ; \vec{\gamma}\right) \times \Gamma \vec{\gamma}
$$

Thus, with $\vec{\gamma}_{I}:=\left(\gamma_{i}\right)_{i \in I}$ and $\Gamma(\vec{r})=\Gamma(\vec{r}, \mathcal{P}):=\prod_{I \in \mathcal{P}} \Gamma\left(\vec{r}_{I}\right) \subseteq \Gamma^{n}$,

$$
\begin{aligned}
\Sigma_{\mathcal{P}}(\vec{r}, \mathbf{k}, \Gamma) & =\bigcup_{\vec{\gamma} \in \Gamma(\vec{r})} \prod_{I \in \mathcal{P}}\left[\Sigma^{\mathrm{nd}}\left(\vec{r}_{I}, \mathbf{k}, \Gamma ; \vec{\gamma}_{I}\right) \times \Gamma \vec{\gamma}_{I}\right] \\
& \subseteq \bigcup_{\vec{\gamma} \in \Gamma(\vec{r})} \prod_{I \in \mathcal{P}}\left[\Sigma\left(\vec{r}_{I}, \mathbf{k}, \Gamma ; \vec{\gamma}_{I}\right) \times \Gamma \vec{\gamma}_{I}\right] .
\end{aligned}
$$

The last product set is contained in $\Sigma(\vec{r}, \mathbf{k}, \Gamma)$ under an obvious identification, so with $\mathcal{P}$ ranging over the partitions of $\{1, \ldots, n\}$, we get

$$
\Sigma(\vec{r}, \mathbf{k}, \Gamma)=\bigcup_{\mathcal{P}} \Sigma_{\mathcal{P}}(\vec{r}, \mathbf{k}, \Gamma)=\bigcup_{\mathcal{P}} \bigcup_{\vec{\gamma} \in \Gamma(\vec{r}, \mathcal{P})} \prod_{I \in \mathcal{P}}\left[\Sigma\left(\vec{r}_{I}, \mathbf{k}, \Gamma ; \vec{\gamma}_{I}\right) \times \Gamma \vec{\gamma}_{I}\right]
$$

This yields the following result.

Lemma 4.2. Suppose $(\mathbf{k}, \Gamma)$ is a Mann pair over $F$, and $\vec{r} \in F^{n}, n \geq 1$. Then $\Sigma(\vec{r}, \mathbf{k}, \Gamma)$ is a finite union of sets $P \times Q$, where $P$ is a $\mathbf{k}$-linear subspace of $\mathbf{k}^{n}$, and $Q \subseteq \Gamma^{n}$ is defined in $\Gamma$ by a conjunction of atoms in the language of $\Gamma$-sets.

Note that the intersection of two sets of the form $P \times Q$, where $P, Q$ are as in Lemma 4.2, is again of the same form. Hence, if $(\mathbf{k}, \Gamma)$ is a Mann pair over $F$, and $\vec{r}, \vec{s} \in F^{n}, n \geq 1$, then $\Sigma(\vec{r}, \mathbf{k}, \Gamma) \cap \Sigma(\vec{s}, \mathbf{k}, \Gamma)$ is a finite union of sets $P \times Q$, where $P, Q$ are as in Lemma 4.2. Next we prove a converse of Lemma 4.2 .

Lemma 4.3. Let $\vec{r} \in \Omega^{n}$. Suppose that

$$
\Sigma(\vec{r}, \mathbf{k}, \Gamma)=\bigcup_{j=1}^{k} P_{j} \times Q_{j},
$$

where $P_{1}, \ldots, P_{k}$ are subsets of $\mathbf{k}^{n}$, and each $Q_{j}$ is a subset of $\Gamma^{n}$ defined in $\Gamma$ by a conjunction of atoms in the language of $\Gamma$-sets. Then there is a finite subset $\Gamma(\vec{r})$ of $\Gamma^{n}$ such that

$$
\Sigma^{\mathrm{nd}}(\vec{r}, \mathbf{k}, \Gamma)=\bigcup_{\vec{\gamma} \in \Gamma(\vec{r})} \Sigma^{\mathrm{nd}}(\vec{r}, \mathbf{k}, \Gamma ; \vec{\gamma}) \times \Gamma \vec{\gamma} .
$$

Proof. We can assume $\Gamma \neq\{1\}$. Let $j \in\{1, \ldots, k\}$ be such that $Q_{j} \neq \emptyset$. By the discussion in [5] just after Corollary 4.2, we can take $\vec{\gamma} \in \Gamma^{n}$ and a partition of $\{1, \ldots, n\}$ into distinct sets $I(1), \ldots, I(l)$ such that

$$
Q_{j}=\Gamma \vec{\gamma}(1) \times \cdots \times \Gamma \vec{\gamma}(l) \subseteq \prod_{\lambda=1}^{l} \Gamma^{I(\lambda)}=\Gamma^{n},
$$

where $\vec{\gamma}(\lambda):=\left\{\left(\gamma_{i}\right)\right\}_{i \in I(\lambda)} \in \Gamma^{I(\lambda)}$ for $\lambda=1, \ldots, l$.

Claim. Let $l>1, \vec{a} \in P_{j}$ and $\vec{\beta} \in Q_{j}$. Then $\left(a_{1} \beta_{1}, \ldots, a_{n} \beta_{n}\right)$ is a degenerate solution of $r_{1} x_{1}+\cdots+r_{n} x_{n}=0$. 
Proof of the claim. For each $\alpha \in \Gamma$,

$$
\sum_{i \in I(1)} r_{i} a_{i} \beta_{i}+\sum_{i \notin I(1)} r_{i} a_{i} \beta_{i}=0=\sum_{i \in I(1)} r_{i} a_{i} \beta_{i}+\sum_{i \notin I(1)} r_{i} a_{i} \alpha \beta_{i} .
$$

So $\sum_{i \notin I(1)} r_{i} a_{i} \beta_{i}=\alpha \sum_{i \notin I(1)} r_{i} a_{i} \beta_{i}$ for all $\alpha \in \Gamma$. Hence $\sum_{i \notin I(1)} r_{i} a_{i} \beta_{i}=0$, and thus $\left(a_{1} \beta_{1}, \ldots, a_{n} \beta_{n}\right)$ is a degenerate solution, proving the claim.

As a result of this claim, we get a subset $J$ of $\{1, \ldots, k\}$, and a tuple $\vec{\gamma}_{j} \in \Gamma^{n}$ for each $j \in J$ such that

$$
\Sigma^{\mathrm{nd}}(\vec{r}, \mathbf{k}, \Gamma) \subseteq \bigcup_{j \in J} P_{j} \times \Gamma \vec{\gamma}_{j}
$$

Since obviously $\bigcup_{j \in J} \Sigma^{\text {nd }}\left(\vec{r}, \mathbf{k}, \Gamma ; \vec{\gamma}_{j}\right) \times \Gamma \vec{\gamma}_{j} \subseteq \Sigma^{\text {nd }}(\vec{r}, \mathbf{k}, \Gamma)$, this yields

$$
\Sigma^{\mathrm{nd}}(\vec{r}, \mathbf{k}, \Gamma)=\bigcup_{j \in J} \Sigma^{\mathrm{nd}}\left(\vec{r}, \mathbf{k}, \Gamma ; \vec{\gamma}_{j}\right) \times \Gamma \vec{\gamma}_{j}
$$

Note: $P_{1}, \ldots, P_{k}$ in this lemma are not assumed to be linear subspaces of $\mathbf{k}^{n}$. Applying the lemma with $\vec{r}=(1, \ldots, 1)$, we obtain

Lemma 4.4. Suppose for each $n \geq 1$ the set $\Sigma_{n}(\mathbf{k}, \Gamma)$ is a finite union of sets $P \times Q$ with $P \subseteq \mathbf{k}^{n}$ and $Q$ a subset of $\Gamma^{n}$ defined in $\Gamma$ by a positive quantifier-free formula in the language of $\Gamma$-sets. Then $(\mathbf{k}, \Gamma)$ is a Mann pair.

Next we improve Lemma 4.4 and obtain a strong version of the implication $(2) \Longrightarrow(1)$ of Theorem 1.2. We shall use facts from [5], but we alert the reader that in the statement and proof of Proposition 5.11 of [5], "subgroups of $G^{n}$ " should be "cosets of subgroups of $G^{n}$ ", and " $B_{n}$ " should be " $B_{m}$ ".

Proposition 4.5. Suppose for each $n \geq 1$ the set $\Sigma_{n}(\mathbf{k}, \Gamma)$ is a finite union of sets $X \times Y$ where $X \subseteq \mathbf{k}^{n}$ and where $Y \subseteq \Gamma^{n}$ is a boolean combination of cosets of subgroups of $\Gamma^{n}$. Then $(\mathbf{k}, \Gamma)$ is a Mann pair.

Proof. Let $n \geq 1$. For $(x, y) \in \mathbf{k}^{n} \times \Gamma^{n}$ we have

$$
x_{1} y_{1}+\cdots+x_{n} y_{n}=1 \Longleftrightarrow\left(\left(x_{1}, \ldots, x_{n},-1\right),\left(y_{1}, \ldots, y_{n}, 1\right)\right) \in \Sigma_{n+1}(\mathbf{k}, \Gamma) .
$$

Using the hypothesis with $n+1$ instead of $n$ it follows easily that the set

$$
\left\{(x, y) \in \mathbf{k}^{n} \times \Gamma^{n}: x_{1} y_{1}+\cdots+x_{n} y_{n}=1\right\}
$$

is a finite union of sets $X \times Y$, where $X \subseteq \mathbf{k}^{n}$ and where $Y \subseteq \Gamma^{n}$ is a boolean combination of cosets of subgroups of $\Gamma^{n}$. Then Proposition 5.11 of [5] yields that $\Gamma$ has the Mann property. Next,

$$
\Sigma_{n}(\mathbf{k}, \Gamma)=\bigcup_{i \in I} X_{i} \times Y_{i}
$$

with finite $I$ and each $X_{i} \subseteq \mathbf{k}^{n}$, and where each $Y_{i} \subseteq \Gamma^{n}$ is a boolean combination of cosets of subgroups of $\Gamma^{n}$. We can also arrange that $X_{i} \neq \emptyset$ for all $i$ and $X_{i} \cap X_{j}=\emptyset$ for all distinct $i, j \in I$.

By Corollary 5.1 and Lemma 5.5 in $\left[\underline{5}\right.$, it follows that each set $Y_{i}$ is definable in $\Gamma$ by a positive quantifier-free formula in the language of $\Gamma$-sets. Then Lemma 4.4 yields that $(\mathbf{k}, \Gamma)$ is a Mann pair. 
Lemma 4.6. If $(\mathbf{k}, \Gamma)$ is a Mann pair, then it is a Mann pair over $\mathbf{k}(\Gamma)$.

Proof. Assume $(\mathbf{k}, \Gamma)$ is a Mann pair, and let $r_{1}, \ldots, r_{n} \in \mathbf{k}(\Gamma)^{\times}$. By Lemma 4.3 . it suffices to prove that $\Sigma(\vec{r}, \mathbf{k}, \Gamma)$ is a finite union of sets $P \times Q$, where $P \subseteq \mathbf{k}^{n}$, and $Q \subseteq \Gamma^{n}$ is defined by a finite conjunction of atoms in the language of $\Gamma$-sets. We may assume that $r_{1}, \ldots, r_{n} \in \mathbf{k}[\Gamma]$. Then $r_{i}=\sum_{j=1}^{k} a_{i j} \beta_{j}$ with $a_{i j} \in \mathbf{k}$, and $\beta_{j} \in \Gamma$ for $i=1, \ldots, n$ and $j=1, \ldots, k$.

Let $\vec{a}=\left(a_{11}, \ldots, a_{1 k}, \ldots, a_{n 1}, \ldots, a_{n k}\right) \in \mathbf{k}^{n k}$. Then

$$
\begin{gathered}
\left(c_{1}, \ldots, c_{n}, \gamma_{1}, \ldots, \gamma_{n}\right) \in \Sigma(\vec{r}, \mathbf{k}, \Gamma) \\
\Longleftrightarrow \\
\left(c_{1}, \ldots, c_{1}, \ldots, c_{n}, \ldots, c_{n}, \beta_{1} \gamma_{1}, \ldots, \beta_{k} \gamma_{1}, \ldots, \beta_{1} \gamma_{n}, \ldots, \beta_{k} \gamma_{n}\right) \in \Sigma(\vec{a}, \mathbf{k}, \Gamma) .
\end{gathered}
$$

By Lemma $4.2 \Sigma(\vec{a}, \mathbf{k}, \Gamma)$ is a finite union of sets $P \times Q$, where $P \subseteq \mathbf{k}^{n k}$, and $Q \subseteq \Gamma^{n k}$ is defined in $\Gamma$ by a conjunction of atoms in the language of $\Gamma$-sets. Fix such $P, Q$, and define

$$
\begin{aligned}
P^{\prime} & :=\left\{\left(c_{1}, \ldots, c_{n}\right) \in \mathbf{k}^{n}:\left(c_{1}, \ldots, c_{1}, \ldots, c_{n}, \ldots, c_{n}\right) \in P\right\}, \\
Q^{\prime} & :=\left\{\left(\gamma_{1}, \ldots, \gamma_{n}\right) \in \Gamma^{n}:\left(\beta_{1} \gamma_{1}, \ldots, \beta_{k} \gamma_{1}, \ldots, \beta_{1} \gamma_{n}, \ldots, \beta_{k} \gamma_{n}\right) \in Q\right\} .
\end{aligned}
$$

Then for $\left(c_{1}, \ldots, c_{n}, \gamma_{1}, \ldots, \gamma_{n}\right) \in \mathbf{k}^{n} \times \Gamma^{n}$,

$$
\begin{gathered}
\left(c_{1}, \ldots, c_{n}, \gamma_{1}, \ldots, \gamma_{n}\right) \in P^{\prime} \times Q^{\prime} \\
\Longleftrightarrow \\
\left(c_{1}, \ldots, c_{1}, \ldots, c_{n}, \ldots, c_{n}, \beta_{1} \gamma_{1}, \ldots, \beta_{k} \gamma_{n}\right) \in P \times Q .
\end{gathered}
$$

Hence, $\Sigma(\vec{a}, \mathbf{k}, \Gamma)$ being a finite union of sets $P \times Q$ with $P$ and $Q$ as above, $\Sigma(\vec{r}, \mathbf{k}, \Gamma)$ is the union of the corresponding sets $P^{\prime} \times Q^{\prime}$. This finishes the proof of the lemma.

Proposition 4.7. If $(\mathbf{k}, \Gamma)$ is a Mann pair, then it is a Mann pair over $\Omega$.

Proof. Let $\vec{r}=\left(r_{1}, \ldots, r_{n}\right) \in \Omega^{n}$. Take a basis $b_{1}, \ldots, b_{m}$ of the $\mathbf{k}(\Gamma)$-linear space $\mathbf{k}(\Gamma) r_{1}+\cdots+\mathbf{k}(\Gamma) r_{n}$. Then $r_{j}=\sum_{i=1}^{m} r_{i j} b_{i}$ with $r_{i j} \in \mathbf{k}(\Gamma)$, so that for all $(\vec{c}, \vec{\gamma}) \in \mathbf{k}^{n} \times \Gamma^{n}$ we have: $(\vec{c}, \vec{\gamma}) \in \Sigma(\vec{r}, \mathbf{k}, \Gamma)$ if and only if $\left(c_{1} \gamma_{1}, \ldots, c_{n} \gamma_{n}\right)$ is a solution of the system

$$
\begin{array}{r}
r_{11} x_{1}+\cdots+r_{1 n} x_{n}=0 \\
r_{21} x_{1}+\cdots+r_{2 n} x_{n}=0 \\
\vdots \\
r_{m 1} x_{1}+\cdots+r_{m n} x_{n}=0 .
\end{array}
$$

Suppose now that $(\mathbf{k}, \Gamma)$ is a Mann pair. Then it is a Mann pair over $\mathbf{k}(\Gamma)$ by the previous lemma. It remains to use Lemmas 4.2 and 4.3

Clearly, this proposition and Lemma 4.2 yield Proposition 4.1 .

For any $n$-tuple $k=\left(k_{1}, \ldots, k_{n}\right) \in \mathbb{Z}^{n}$, consider the character

$$
\chi_{k}:\left(\Omega^{\times}\right)^{n} \rightarrow \Omega^{\times}, \quad \chi_{k}\left(y_{1}, \ldots, y_{n}\right):=y_{1}^{k_{1}} \cdots y_{n}^{k_{n}} .
$$

This is a multiplicative group homomorphism. For any $e \in \mathbb{N}$, let $\mathcal{D}(n, e)$ be the finite collection of subgroups of $\left(\Omega^{\times}\right)^{n}$ that are intersections of kernels of characters $\chi_{k}$ with $|k|=\left|k_{1}\right|+\cdots+\left|k_{n}\right| \leq e$. 
Proposition 4.8. Let $(\mathbf{k}, \Gamma)$ be a Mann pair, let $X_{1}, \ldots, X_{m}, Y_{1}, \ldots, Y_{n}$ be distinct indeterminates, and let the polynomials $f_{1}, \ldots, f_{M} \in \Omega[X, Y]$ have degree $\leq d$ in $X=\left(X_{1}, \ldots, X_{m}\right)$ and degree $\leq e$ in $Y=\left(Y_{1}, \ldots, Y_{n}\right)$, where $M, d, e \in \mathbb{N}$, and put

$$
Z:=\left\{(x, y) \in \mathbf{k}^{m} \times \Gamma^{n}: f_{1}(x, y)=\cdots=f_{M}(x, y)=0\right\} .
$$

Then $Z$ is a finite union of sets $P \times Q$, where

$$
P=\left\{x \in \mathbf{k}^{m}: g_{1}(x)=\cdots=g_{N}(x)=0\right\}
$$

for suitable $N \in \mathbb{N}$ and polynomials $g_{1}, \ldots, g_{N} \in \mathbf{k}[X]$ of degree $\leq d$, and where $Q \subseteq \Gamma^{n}$ is a coset of a subgroup $D \cap \Gamma^{n}$ of $\Gamma^{n}$ with $D \in \mathcal{D}(n, e)$.

Proof. The intersection of finitely many cosets of such subgroups is either empty or again a coset of such a subgroup. Hence we may (and shall) assume that $M=1$. Put $f:=f_{1}$. Then $f=\sum_{(i, j) \in I \times J} a_{i j} X^{i} Y^{j}$ where all $a_{i j} \in \Omega$ and $I$ is the set of multi-indices $i=\left(i_{1}, \ldots, i_{m}\right) \in \mathbb{N}^{m}$ with $|i|=i_{1}+\cdots+i_{m} \leq d$ and $J$ is the set of multi-indices $j=\left(j_{1}, \ldots, j_{n}\right) \in \mathbb{N}^{n}$ with $|j|=j_{1}+\cdots+j_{n} \leq e$. By Proposition 4.7 and Lemma 4.2 the set

$$
\left\{\left(\left(x_{i}\right)_{i \in I},\left(y_{j}\right)_{j \in J}\right) \in \mathbf{k}^{I} \times \Gamma^{J}: \sum_{(i, j) \in I \times J} a_{i j} x_{i} y_{j}=0\right\}
$$

is a finite union of subsets of $\mathbf{k}^{I} \times \Gamma^{J}$ of the form

$$
V \times\left\{y \in \Gamma^{J}: \gamma_{1} y_{i(1)}=y_{j(1)}, \ldots, \gamma_{k} y_{i(k)}=y_{j(k)}\right\}
$$

with $V$ a k-linear subspace of $\mathbf{k}^{I}, \gamma_{1}, \ldots, \gamma_{k} \in \Gamma$, and with $k \in \mathbb{N}$ and indices $i(1), j(1) \ldots, i(k), j(k)$ in $J$. It remains to observe that for such $\gamma_{1}, \ldots, \gamma_{k}$ and $i(1), j(1), \ldots, i(k), j(k)$ the set

$$
\left\{y \in \Gamma^{J}: \gamma_{1} \chi_{i(1)}(y)=\chi_{j(1)}(y), \ldots, \gamma_{k} \chi_{i(k)}(y)=\chi_{j(k)}(y)\right\}
$$

is a coset of the subgroup $D \cap \Gamma^{n}$ of $\Gamma^{n}$ where $D$ is the intersection of the kernels of $\chi_{i(1)-j(1)}, \ldots, \chi_{i(k)-j(k)}$.

\section{Robustness of Mann pairs}

In this section we assume that $(\mathbf{k}, \Gamma)$ is a Mann pair. In addition, $K \supseteq \mathbf{k}$ is a subfield of $\Omega$, and $\Delta, \Gamma^{\prime}$ are subgroups of $\Omega^{\times}$with $\Gamma^{\prime} \supseteq \Gamma$. We claim:

(1) $[K: \mathbf{k}]<\infty \Rightarrow(K, \Gamma)$ is a Mann pair;

(2) $\left[\Gamma^{\prime}: \Gamma\right]<\infty \Rightarrow\left(\mathbf{k}, \Gamma^{\prime}\right)$ is a Mann pair;

(3) $(K \supseteq \mathbf{k}(\Gamma)$ and $(K, \Delta)$ is a Mann pair $) \Rightarrow(\mathbf{k}, \Gamma \Delta)$ is a Mann pair;

(4) ( $K$ is linearly disjoint from $\mathbf{k}(\Gamma)$ over $\mathbf{k}) \Rightarrow(K, \Gamma)$ is a Mann pair.

Proof of (1). Here $K$ is an extension field of finite degree $m$ over k. Let $b_{1}, \ldots, b_{m}$ be a basis of the $\mathbf{k}$-linear space $K$. Let $n \geq 1$, and consider $(\vec{a}, \vec{\gamma}) \in K^{n} \times \Gamma^{n}$. Then with $a_{j}=\sum_{i=1}^{m} a_{i j} b_{i}\left(1 \leq j \leq n\right.$, all $\left.a_{i j} \in \mathbf{k}\right)$,

$$
\begin{aligned}
(\vec{a}, \vec{\gamma}) \in \Sigma_{n}(K, \Gamma) & \Longleftrightarrow \sum_{j=1}^{n}\left(a_{1 j} b_{1}+\cdots+a_{m j} b_{m}\right) \gamma_{j}=0 \\
& \Longleftrightarrow F\left(a_{11}, \ldots, a_{m n}, \gamma_{1}, \ldots, \gamma_{n}\right)=0
\end{aligned}
$$

with $F:=\sum_{j=1}^{n}\left(X_{1 j} b_{1}+\cdots+X_{m j} b_{m}\right) Y_{j} \in K\left[X_{11}, \ldots, X_{m n}, Y_{1}, \ldots, Y_{n}\right]$. 
By Proposition 4.8 , the set

$$
\left\{\left(\left(c_{i j}\right), \gamma\right) \in \mathbf{k}^{m n} \times \Gamma^{n}: F\left(c_{11}, \ldots, c_{m n}, \gamma_{1}, \ldots, \gamma_{n}\right)=0\right\}
$$

is a union $\bigcup_{i \in I} P_{i} \times Q_{i}$ with finite $I$, where each $P_{i} \subseteq \mathbf{k}^{m n}$, and each $Q_{i} \subseteq \Gamma^{n}$ is a coset of a subgroup of $\Gamma^{n}$. Therefore we have $\Sigma_{n}(K, \Gamma)=\bigcup_{i \in I} P_{i}^{\prime} \times Q_{i}$ with $P_{i}^{\prime}=\left\{\vec{a} \in K^{n}:\left(a_{i j}\right) \in P_{i}\right\}$, where $\vec{a}$ determines $\left(a_{i j}\right)$ as above.

Corollary 5.1. If $\gamma \in \Gamma$ is algebraic over $\mathbf{k}$, then $\gamma$ is a root of unity. In particular, if $(\mathbb{Q}, \Gamma)$ is a Mann pair with $\Gamma \subseteq A^{\times}$, then $\Gamma \subseteq \mathbb{U}$.

Proof of (2). Assume $\Gamma^{\prime}=\bigcup_{\alpha \in C} \alpha \Gamma$ where $C \subseteq \Gamma^{\prime}$ is finite. Then

$$
\Sigma_{n}\left(\mathbf{k}, \Gamma^{\prime}\right)=\bigcup_{\vec{\alpha} \in C^{n}}\{(\vec{a}, \vec{\alpha} \vec{\gamma}):(\vec{a}, \vec{\gamma}) \in \Sigma(\vec{\alpha}, \mathbf{k}, \Gamma)
$$

and it remains to use Proposition 4.7, Lemma 4.2 and Proposition 4.5.

Proof of (3). Assume $K \supseteq \mathbf{k}(\Gamma)$ and $(K, \Delta)$ is a Mann pair. Let $n \geq 1$ and take a finite $\Delta(n) \subseteq \Delta^{n}$ such that

$$
\Sigma_{n}^{\mathrm{nd}}(K, \Delta)=\bigcup_{\vec{\delta} \in \Delta(n)} \Sigma_{n}^{\mathrm{nd}}(K, \Delta ; \vec{\delta}) \times \Delta \vec{\delta} .
$$

For each $\vec{\delta} \in \Delta(n)$ we have a finite $\Gamma(\vec{\delta}) \subseteq \Gamma^{n}$ such that

$$
\Sigma^{\mathrm{nd}}(\vec{\delta}, \mathbf{k}, \Gamma)=\bigcup_{\vec{\gamma} \in \Gamma(\vec{\delta})} \Sigma^{\mathrm{nd}}(\vec{\delta}, \mathbf{k}, \Gamma ; \vec{\gamma}) \times \Gamma \vec{\gamma} .
$$

It follows that, with $\vec{\gamma} \vec{\delta}:=\left(\gamma_{1} \delta_{1}, \ldots, \gamma_{n} \delta_{n}\right)$, we have

$$
\Sigma_{n}^{\mathrm{nd}}(\mathbf{k}, \Gamma \Delta)=\bigcup_{\vec{\delta} \in \Delta(n)} \bigcup_{\vec{\gamma} \in \Gamma(\vec{\delta})} \Sigma_{n}^{\mathrm{nd}}(\mathbf{k}, \Gamma \Delta ; \vec{\gamma} \vec{\delta}) \times \Gamma \Delta \vec{\gamma} \vec{\delta} .
$$

Proof of (4). Assume $K$ is linearly disjoint from $\mathbf{k}(\Gamma)$ over $\mathbf{k}$. Let $n \geq 1$; it is enough to obtain $\Sigma_{n}(K, \Gamma)$ as a finite union of sets $P \times Q$ with $P \subseteq K^{n}$, and $Q \subseteq \Gamma^{n}$ defined in $\Gamma$ by a conjunction of atoms in the language of $\Gamma$-sets.

Suppose $(\vec{r}, \vec{\gamma}) \in \Sigma_{n}(K, \Gamma)$. Then take a basis $b_{1}, \ldots, b_{m}$ of the $\mathbf{k}$-linear space $\mathbf{k} r_{1}+\cdots+\mathbf{k} r_{n}$. Then $r_{j}=\sum_{i=1}^{m} a_{i j} b_{i}$ with $a_{i j} \in \mathbf{k}, j=1, \ldots, n$; hence

$$
\left(a_{i 1}, \ldots, a_{i n}, \vec{\gamma}\right) \in \Sigma_{n}(\mathbf{k}, \Gamma) \text { for } i=1, \ldots, m .
$$

Conversely, given any $a_{i j} \in \mathbf{k}(1 \leq i, j \leq n)$ such that

$$
\left(a_{i 1}, \ldots, a_{i n}, \vec{\gamma}\right) \in \Sigma_{n}(\mathbf{k}, \Gamma) \text { for } i=1, \ldots, n
$$

and any $b_{1}, \ldots, b_{n} \in K$ (not necessarily linearly independent over $\mathbf{k}$ ), we have $(\vec{r}, \vec{\gamma}) \in \Sigma_{n}(K, \Gamma)$, where $r_{j}=\sum_{i=1}^{n} a_{i j} b_{i}$ for $j=1, \ldots, n$. It follows that for all $(\vec{r}, \vec{\gamma}) \in K^{n} \times \Gamma^{n}$ we have: $(\vec{r}, \vec{\gamma}) \in \Sigma_{n}(K, \Gamma)$ if and only if there is an $n \times n$ matrix $A=\left(a_{i j}\right) \in \mathbf{k}^{n^{2}}$ and a vector $\vec{b} \in K^{n}$ such that

$$
\left(a_{i 1}, \ldots, a_{i n}, \vec{\gamma}\right) \in \Sigma_{n}(\mathbf{k}, \Gamma) \text { for } i=1, \ldots, n \text {, and } A \vec{b}=\vec{r},
$$

with $\vec{b}$ and $\vec{r}$ viewed as column vectors. By Lemma 4.2 the set of all $(A, \vec{\gamma}) \in$ $\mathbf{k}^{n^{2}} \times \Gamma^{n}$, with $A=\left(a_{i j}\right)$, such that the displayed condition holds, is a union $\bigcup_{\lambda \in \Lambda} P_{\lambda} \times Q_{\lambda}$ with finite $\Lambda$ where each $P_{\lambda} \subseteq \mathbf{k}^{n^{2}}$, and each $Q_{\lambda} \subseteq \Gamma^{n}$ is defined in $\Gamma$ by a conjunction of atoms in the language of $\Gamma$-sets. 
For $\lambda \in \Lambda$, let $P_{\lambda}^{\prime}$ be the set of all $\vec{r} \in K^{n}$ for which there is a matrix $A \in P_{\lambda}$ and a vector $\vec{b} \in K^{n}$ such that $A \vec{b}=\vec{r}$. Then by the above, $\Sigma_{n}(K, \Gamma)=\bigcup_{\lambda \in \Lambda} P_{\lambda}^{\prime} \times Q_{\lambda}$.

5.1. Curious Mann pairs in positive characteristic. Let $p$ be a prime number, and let $\Omega \supseteq \mathbb{F}_{p}$ be of characteristic $p$ so that every $\mathbb{F}_{p^{e}}$ with $e$ a positive integer is a subfield of $\Omega$. Let $P$ be a finite nonempty set of prime numbers different from $p$, let $S(P)$ be the set of all positive integers all of whose prime factors are in $P$. Put

$$
G:=\left\{a \in \Omega^{\times}: a^{N}=1 \text { for some } N \in S(P)\right\},
$$

an infinite subgroup of $\Omega^{\times}$isomorphic to the direct product of the Prüfer groups $\mathbb{Z}\left(l^{\infty}\right)$ with $l \in P$.

Theorem 8.9 in 5 says that $G$ has the Mann property, but this is also a consequence of earlier results in [2] and [15]. We now strengthen this as follows. The proof in [5] starts with a certain large enough finite field $\mathbb{F}_{p^{e}}$ where $e$ is a positive integer. Put

$$
f:=e \cdot \prod_{l \notin P} l^{\infty}, \quad g:=e \cdot \prod_{l \in P} l^{\infty} \quad \text { (products of supernatural numbers). }
$$

Then $\mathbb{F}_{p^{e}}(G)=\mathbb{F}_{p^{g}} \subseteq \Omega$, and so the infinite field $\mathbb{F}_{p^{f}}$ is linearly disjoint from $\mathbb{F}_{p^{e}}(G)$ over $\mathbb{F}_{p^{e}}$, so by (4) above we have a Mann pair $\left(\mathbb{F}_{p^{f}}, G\right)$ with the curious property that $\mathbb{F}_{p^{f}}(G)=\mathbb{F}_{p}^{\text {ac }}$.

5.2. Some Mann pairs in mixed characteristic. Let $p$ be a prime number and $E \supseteq \mathbb{F}_{p}$ a perfect field of characteristic $p$. Let $W[E] \supseteq W\left[\mathbb{F}_{p}\right]=\mathbb{Z}_{p}$ be the ring of Witt vectors over $E$, let $W(E) \supseteq W\left(\mathbb{F}_{p}\right)=\mathbb{Q}_{p}$ be its fraction field, and let $\tau: E^{\times} \rightarrow W(E)^{\times}$be the Teichmüller lifting. It was shown in [5], 8.4, that $\tau\left(E^{\times}\right)$ satisfies a strong form of the Mann property. In fact:

Proposition 5.2. $\left(\mathbb{Q}_{p}, \tau\left(E^{\times}\right)\right)$is a Mann pair.

Proof. Consider first the case $E=\mathbb{F}_{p}^{a c}$. Then

$$
\tau\left(E^{\times}\right)=\mathbb{U}\left[p^{\prime}\right]:=\left\{x \in W(E): x^{n}=1 \text { for some } n \text { not divisible by } p\right\} .
$$

By arguments as in the proof of Theorem 1 in $\left[12\right.$ one shows that $\left(\mathbb{Q}_{p}, \mathbb{U}\left[p^{\prime}\right]\right)$ is a Mann pair. (Alternatively, $\left(\mathbb{Q}, \mathbb{U}\left[p^{\prime}\right]\right)$ is a Mann pair by Theorem 1 in [12], and it remains to use $(4)$ and the fact that $\mathbb{Q}_{p}$ and $\mathbb{Q}\left(\mathbb{U}\left[p^{\prime}\right]\right)$ are linearly disjoint over $\mathbb{Q}$.)

In the general case, we first arrange that $E$ is algebraically closed so that $E \supseteq \mathbb{F}_{p}^{\text {ac }}$ and $W(E) \supseteq W\left(\mathbb{F}_{p}^{\text {ac }}\right)$. Then $E^{\times}=\left(\mathbb{F}_{p}^{\text {ac }}\right)^{\times} G$, where the subgroup $G$ of $E^{\times}$is torsion-free. Then $\tau\left(E^{\times}\right)=\mathbb{U}\left[p^{\prime}\right] \tau(G)$. By the proof of 8.4 in [5], whenever $\gamma_{1}, \ldots, \gamma_{n} \in \tau(G)$ are multiplicatively independent, they are algebraically independent over $W\left(\mathbb{F}_{p}^{\mathrm{ac}}\right)$. Then Lemma 2.3 yields that $\left(W\left(\mathbb{F}_{p}^{\mathrm{ac}}\right), \tau(G)\right)$ is a Mann pair. Since $\left(\mathbb{Q}_{p}, \mathbb{U}\left[p^{\prime}\right]\right)$ is a Mann pair, we obtain from $(3)$ that $\left(\mathbb{Q}_{p}, \tau\left(E^{\times}\right)\right)$is a Mann pair.

\section{Proof of Theorem 1.1}

In this section we assume that $\Omega$ has characteristic zero, and, besides $\mathbf{k}$, we also let $E, F, K$ be subfields of $\Omega$. The following contains Proposition 5.16 of [5] as a special case, with almost the same proof. 
Lemma 6.1. Let $E$ be a subfield of $K$ such that all $p^{\text {th }}$ roots of unity in $K$ are in $E$, for every prime $p$. Let $G$ be a pure subgroup of $E^{\times}$, and put

$$
H:=\left\{h \in K^{\times}: h^{d} \in G \text { for some positive integer } d\right\} .
$$

Then for $a_{1}, \ldots, a_{n} \in E^{\times}$, the equation $a_{1} x_{1}+\cdots+a_{n} x_{n}=1$ has the same nondegenerate solutions in $G$ as in $H$.

Proof. By Lemma 5.15 of [5], it is enough to show the following.

Claim. Let $p$ be a prime number, and let $h \in K^{\times}$be such that $h^{p}=g \in G$ and $h \notin G$. Then $X^{p}-g \in E[X]$ is irreducible, and $G h^{\mathbb{Z}}$, the subgroup of $K^{\times}$generated by $G$ and $h$, is pure in $E(h)^{\times}$.

Proof of the Claim. Since $h \notin G$ and $K$ and $E$ have the same $p^{\text {th }}$ roots of unity, $g$ is not a $p^{\text {th }}$ power in $E^{\times}$. Thus by Theorem 9.1 from [9] the polynomial $X^{p}-g$ is irreducible in $E[X]$. To show that $G h^{\mathbb{Z}}=\bigcup_{i=0}^{p-1} G h^{i}$ is pure in $E(h)^{\times}$, suppose towards a contradiction that $f \in E(h)^{\times}$and $f^{d} \in G h^{\mathbb{Z}}$, where $d$ is an integer greater than 1 , but $f \notin G h^{\mathbb{Z}}$. We can reduce to the case that $d$ is prime and $f^{m} \notin G$ for $1 \leq m<d$. So by Theorem 9.1 of [9] again, $X^{d}-f^{d}$ is irreducible in $E[X]$. As $E(f) \subseteq E(h)$ and $[E(h): E]=p$, we get $d=p$. Let $\zeta$ be a primitive $p^{\text {th }}$ root of unity in the algebraic closure of $K$. Then $E(h) \cap E(\zeta)=E$ and $E(h, \zeta)$ is a cyclic extension of degree $p$ of $E(\zeta)$. Let $\sigma \in \operatorname{Gal}(E(h, \zeta) \mid E(\zeta))$ be given by $\sigma(h)=\zeta h$. Then $\sigma(f)=\zeta^{k} f$ with $0<k<p$. With $f=c_{0}+c_{1} h+\cdots+c_{p-1} h^{p-1}$ and all $c_{i} \in E$ this gives

$$
\begin{aligned}
\sigma(f) & =c_{0}+\zeta c_{1} h+\cdots+\zeta^{p-1} c_{p-1} h^{p-1} \\
& =\zeta^{k} c_{0}+\zeta^{k} c_{1} h+\cdots+\zeta^{k} c_{p-1} h^{p-1} .
\end{aligned}
$$

This forces $c_{i}=0$ for all $i \neq k$, so $f=c_{k} h^{k}$, as desired.

Lemma 6.2. Suppose $\mathbf{k}$ is algebraically closed, $F \supseteq \mathbf{k}, \operatorname{trdeg}(F \mid \mathbf{k})=1, \mathbf{k}^{\times} \cap \Gamma=$ $\{1\}$, and $\Gamma \subseteq F^{\times}$has finite rank. Then $(\mathbf{k}, \Gamma)$ is a Mann pair.

Proof. We can assume that $\Gamma \neq\{1\}$. Consider the divisible closure

$$
H:=\left\{f \in F^{\times}: f^{d} \in \mathbf{k}^{\times} \Gamma \text { for some positive integer } d\right\},
$$

of $\mathbf{k}^{\times} \Gamma$ in $F^{\times}$. Now, as an abelian group, $\mathbf{k}^{\times}$is divisible and hence injective, so we can take a subgroup $\Gamma^{\prime} \supseteq \Gamma$ of $H$ such that $\mathbf{k}^{\times} \cap \Gamma^{\prime}=\{1\}$ and $\mathbf{k}^{\times} \Gamma^{\prime}=H$. It follows that $\Gamma^{\prime}$ is pure in $F^{\times}$and $\Gamma^{\prime} / \Gamma$ is a torsion group. Replacing $\Gamma$ by $\Gamma^{\prime}$ we arrange that $\Gamma$ is pure in $F^{\times}$. Take a finitely generated subgroup $\Gamma_{0}$ of $\Gamma$ such that $\Gamma / \Gamma_{0}$ is a torsion group. Then $E:=\mathbf{k}\left(\Gamma_{0}\right)$ is a function field over $\mathbf{k}$ of one variable. Take a finite subset $S$ of $\mathcal{R}(E \mid \mathbf{k})$ such that all elements of $\Gamma_{0}$ are $S$-units, and put $\Gamma_{1}:=E^{\times} \cap \Gamma$. Since $\Gamma_{1} / \Gamma_{0}$ is a torsion group, all elements of $\Gamma_{1}$ are also $S$-units. Hence $\Gamma_{1}$ is isomorphic to a subgroup of $\bigoplus_{v \in S} \mathbb{Z} v$, and is thus finitely generated. Then by Corollary 3.2. $\left(\mathbf{k}, \Gamma_{1}\right)$ is a Mann pair. Since $\Gamma$ is pure in $F^{\times}$and $\mathbf{k}$ contains all roots of unity, $\Gamma_{1}$ is pure in $E^{\times}$. Now apply Lemmas 6.1 and 2.1 to get that $(\mathbf{k}, \Gamma)$ is a Mann pair.

Theorem 6.3. Suppose $\mathbf{k}$ is algebraically closed, $\mathbf{k}^{\times} \cap \Gamma=\{1\}$, and $\Gamma$ has finite rank. Then $(\mathbf{k}, \Gamma)$ is a Mann pair. 
Proof. Let $F$ be the algebraic closure of $\mathbf{k}(\Gamma)$ in $\Omega$, and put

$$
H:=\left\{f \in F^{\times}: f^{d} \in \mathbf{k}^{\times} \Gamma \text { for some positive integer } d\right\},
$$

the divisible closure of $\mathbf{k}^{\times} \Gamma$ in $F^{\times}$. Now, as an abelian group, $\mathbf{k}^{\times}$is divisible and hence injective, so we can take a subgroup $\Gamma^{\prime} \supseteq \Gamma$ of $H$ such that $\mathbf{k}^{\times} \cap \Gamma^{\prime}=\{1\}$ and $\mathbf{k}^{\times} \Gamma^{\prime}=H$. It follows that $\Gamma^{\prime}$ is divisible and $\Gamma^{\prime} / \Gamma$ is a torsion group. So replacing $\Gamma$ by $\Gamma^{\prime}$ if necessary we can assume in the rest of the proof that $\Gamma$ is divisible. Let $m:=\operatorname{trdeg}(\mathbf{k}(\Gamma) \mid \mathbf{k})$, and take a chain

$$
\mathbf{k}=K_{0} \subseteq K_{1} \subseteq \cdots \subseteq K_{m}=F
$$

of algebraically closed subfields of $\Omega$ such that for $i=0, \ldots, m-1$ we have $\operatorname{trdeg}\left(K_{i+1} \mid K_{i}\right)=1$. Put

$$
\Gamma_{i}:=\Gamma \cap K_{i}^{\times}, \quad i=1, \ldots, m .
$$

We claim that $\left(\mathbf{k}, \Gamma_{i}\right)$ is a Mann pair for $i=1, \ldots, m$. For $i=1$ this holds by Lemma 6.2. since $\mathbf{k}\left(\Gamma_{1}\right) \subseteq K_{1}$ and $K_{1}$ has transcendence degree 1 over $\mathbf{k}$. Suppose $1 \leq i<m$ and $\left(\mathbf{k}, \Gamma_{i}\right)$ is a Mann pair. Since $\Gamma_{i}$ is divisible we have a subgroup $\Delta$ of $\Gamma_{i+1}$ such that $\Gamma_{i+1}=\Gamma_{i} \Delta$ and $\Gamma_{i} \cap \Delta=\{1\}$. Then $\Delta$ has finite rank and $K_{i}^{\times} \cap \Delta=\{1\}$, and $K_{i}(\Delta) \subseteq K_{i+1}$, so $\left(K_{i}, \Delta\right)$ is a Mann pair. It follows from (3) in Section 5 that then $\left(\mathbf{k}, \Gamma_{i} \Delta\right)=\left(\mathbf{k}, \Gamma_{i+1}\right)$ is a Mann pair.

This finishes the inductive proof of the claim. For $i=m$ this claim yields the desired conclusion.

In this theorem we cannot replace the finite rank assumption by the weaker condition that $\Gamma$ has the Mann property, as the following example shows. Take distinct indeterminates $a_{1}, x_{1}, a_{2}, x_{2}, a_{3}, x_{3}, \ldots$ and put

$$
\mathbf{k}:=\mathbb{Q}\left(a_{1}, a_{2}, a_{3}, \ldots\right)^{\mathrm{ac}}, \quad K:=\mathbf{k}\left(x_{1}, x_{2}, x_{2}, \ldots\right) .
$$

Let $\Gamma$ be the subgroup of $K^{\times}$generated by $x_{1}, y_{1}, x_{2}, y_{2}, x_{3}, y_{3}, \ldots$, where $y_{i}:=$ $1-a_{i} x_{i}$. Then $\mathbf{k}$ is algebraically closed, $\mathbf{k}^{\times} \cap \Gamma=\{1\}$, but $(\mathbf{k}, \Gamma)$ is not a Mann pair, since $a_{i} x_{i}+y_{i}=1$ for all $i$. On the other hand, $\Gamma$ has the Mann property because $x_{1}, y_{1}, x_{2}, y_{2}, \ldots$ are algebraically independent over $\mathbb{Q}$ (but not over $\mathbf{k}$ ).

The condition $\mathbf{k}^{\times} \cap \Gamma=\{1\}$ in the theorem can be relaxed:

Corollary 6.4. Suppose $\mathbf{k}$ is algebraically closed, $\mathbf{k}^{\times} \cap \Gamma$ is finite and $\Gamma$ has finite rank. Then $(\mathbf{k}, \Gamma)$ is a Mann pair.

Proof. Put $d:=\left|\mathbf{k}^{\times} \cap \Gamma\right|$. The hypothesis implies that then the subgroup $\Delta:=$ $\left\{\gamma^{d}: \gamma \in \Gamma\right\}$ of $\Gamma$ is of finite rank and has finite index in $\Gamma$, and that $\mathbf{k}^{\times} \cap \Delta=\{1\}$. Hence by the theorem, $\left(\mathbf{k}^{\mathrm{ac}}, \Delta\right)$ is a Mann pair, and thus $(\mathbf{k}, \Gamma)$ is a Mann pair by item (2) of the previous section.

Another proof. The referee mentioned that Theorem 6.3 can be obtained from [7]. Here is a sketch of how. With the assumptions of Theorem 6.3 we can arrange in addition that $\Omega$ is equipped with a derivation making it a differentially closed field with $\mathbf{k}$ as its field of constants. Below, "definable", "Morley rank", and "Morley degree" are with respect to $\Omega^{\mathrm{eq}}$, with $\Omega$ viewed as a differentially closed field. The derivation of $\Omega$ yields the logarithmic derivative map ld as a definable group morphism

$$
x \mapsto x^{\prime} / x: \mathbb{G}_{\mathrm{m}}=\Omega^{\times} \rightarrow \mathbb{G}_{\mathrm{a}}=\Omega,
$$


with kernel $\mathbf{k}^{\times}$. Let $\gamma_{1}, \ldots, \gamma_{r} \in \Gamma$ with $r \in \mathbb{N}$ generate a subgroup $\Gamma_{0}$ such that $\Gamma / \Gamma_{0}$ is a torsion group. Then

$$
\operatorname{ld}(\Gamma) \subseteq \mathbb{Q} \cdot \operatorname{ld}\left(\gamma_{1}\right)+\cdots+\mathbb{Q} \cdot \operatorname{ld}\left(\gamma_{r}\right) \subseteq \mathbf{k} \cdot \operatorname{ld}\left(\gamma_{1}\right)+\cdots+\mathbf{k} \cdot \operatorname{ld}\left(\gamma_{r}\right)=: V
$$

Put $G:=\mathrm{ld}^{-1}(V)$, a definable subgroup of $\mathbb{G}_{\mathrm{m}}$ of finite Morley rank with $\mathbf{k}^{\times} \subseteq G$ and $\Gamma \subseteq G$. Let $n \geq 2$ and let $X \subseteq G^{n}$ be the set of nondegenerate solutions of the equation $x_{1}+\cdots+x_{n}=0$ in $G$; by Lemma 2.1 it is enough to obtain a finite set $F(n) \subseteq G^{n}$ such that

$$
X \subseteq\left\{\left(c_{1} g_{1}, \ldots, c_{n} g_{n}\right): c_{1}, \ldots, c_{n} \in \mathbf{k}^{\times},\left(g_{1}, \ldots, g_{n}\right) \in F(n)\right\} .
$$

The semiabelian variety $\mathbb{G}_{\mathrm{m}}^{n}$ and its diagonal algebraic subgroup $\Delta:=\{(g, \ldots, g)$ : $\left.g \in \mathbb{G}_{\mathrm{m}}\right\}$ are defined over $\mathbf{k}$, which gives the semiabelian variety $A:=\mathbb{G}_{\mathrm{m}}^{n} / \Delta$ over $\mathbf{k}$. Let $\bar{G} \subseteq A$ be the image of $G^{n}$ under the natural map $\mathbb{G}_{\mathrm{m}}^{n} \rightarrow A$, so $\bar{G}$ is a definable subgroup of $A$ of finite Morley rank containing $A(\mathbf{k})$. For $x \in G^{n}$, let $\bar{x}$ be its image in $\bar{G}$, and let $\bar{X}:=\{\bar{x}: x \in X\} \subseteq \bar{G}$. It is now an exercise to show that each definable subset of $\bar{X}$ of Morley degree 1 has trivial model-theoretic stabilizer in $\bar{G}$, as defined in [7. This allows us to apply Corollary 2.7 of [7] inductively to obtain that $\bar{X}$ is covered by finitely many cosets of $A(\mathbf{k})$; this gives the finite set $F(n) \subseteq G^{n}$, as desired.

This proof does not seem to have the same kind of effectiveness as the proof based on the Brownawell-Masser height bound.

\section{Definable Sets in Mann Pairs}

Let $\mathcal{L}$ be the language of rings augmented by two distinct unary relation symbols. Let $T$ be the $\mathcal{L}$-theory whose models are the structures $(\Omega, \mathbf{k}, \Gamma)$ where $\Omega$ is an algebraically closed field with a subfield $\mathbf{k}$, and a subgroup $\Gamma$ of $\Omega^{\times}$. Let $\mathcal{L}_{\Sigma}^{\mathrm{f}, \mathrm{g}}$ be the 2-sorted language, with sorts f,g (the field sort and the group sort), and with the following nonlogical symbols:

- constant symbols 0 and 1 of sort $f$,

- a unary function symbol - of sort $(f ; f)$,

- binary function symbols + and · of sort $(f, f ; f)$,

- a constant symbol 1 of sort g,

- a unary function symbol ${ }^{-1}$ of sort $(\mathrm{g} ; \mathrm{g})$,

- a binary function symbol - of sort (g,g;g),

- for each $n \geq 1$, a $2 n$-ary relation symbol $\Sigma_{n}$ of sort (f, $\left.\ldots, \mathrm{f}, \mathrm{g}, \ldots, \mathrm{g}\right)(n$ places of sort $\mathrm{f}$ and $n$ places of sort $\mathrm{g}$ ).

Of course, "1 used as a symbol of sort f" is different from " 1 used as a symbol of sort g" and likewise with the multiplication symbol. For a model $(\Omega, \mathbf{k}, \Gamma)$ of $T$ we construe $(\mathbf{k}, \Gamma)$ as an $\mathcal{L}_{\Sigma}^{\mathrm{f}, \mathrm{g}}$-structure by interpreting the symbols in the obvious way; in particular, each $\Sigma_{n}$ is interpreted as the previously defined $\Sigma_{n}(\mathbf{k}, \Gamma) \subseteq \mathbf{k}^{n} \times \Gamma^{n}$.

In the next result we do not assume that $(\mathbf{k}, \Gamma)$ is a Mann pair.

Lemma 7.1. Let $(\Omega, \mathbf{k}, \Gamma) \models T$. Then every subset of $\mathbf{k}^{m} \times \Gamma^{n}$ definable in $(\Omega, \mathbf{k}, \Gamma)$ is definable in the $\mathcal{L}_{\Sigma}^{\mathrm{f}, \mathrm{g}}$-structure $(\mathbf{k}, \Gamma)$. 
Proof. Take an $|\Omega|^{+}$-saturated elementary extension $\left(\Omega^{\prime}, \mathbf{k}^{\prime}, \Gamma^{\prime}\right)$ of $(\Omega, \mathbf{k}, \Gamma)$. It is easy to check that then $\mathbf{k}^{\prime}\left(\Gamma^{\prime}\right)$ and $\Omega$ are linearly disjoint over $\mathbf{k}(\Gamma)$ :

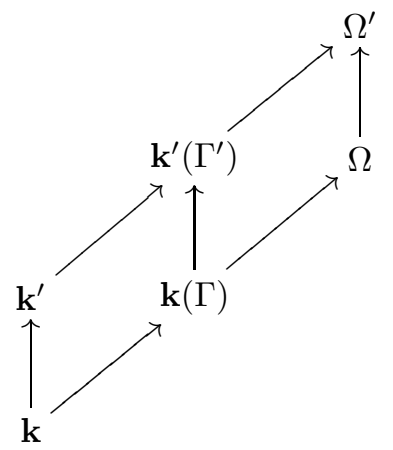

Let $\vec{a}, \vec{b} \in\left(\mathbf{k}^{\prime}\right)^{m}$ and $\vec{\alpha}, \vec{\beta} \in\left(\Gamma^{\prime}\right)^{n}$ be such that

$$
\operatorname{tp}_{\left(\mathbf{k}^{\prime}, \Gamma^{\prime}\right)}((\vec{a}, \vec{\alpha}) \mid(\mathbf{k}, \Gamma))=\operatorname{tp}_{\left(\mathbf{k}^{\prime}, \Gamma^{\prime}\right)}((\vec{b}, \vec{\beta}) \mid(\mathbf{k}, \Gamma)) .
$$

It suffices to prove that then

$$
\operatorname{tp}_{\left(\Omega^{\prime}, \mathbf{k}^{\prime}, \Gamma^{\prime}\right)}((\vec{a}, \vec{\alpha}) \mid(\Omega, \mathbf{k}, \Gamma))=\operatorname{tp}_{\left(\Omega^{\prime}, \mathbf{k}^{\prime}, \Gamma^{\prime}\right)}((\vec{b}, \vec{\beta}) \mid(\Omega, \mathbf{k}, \Gamma)) .
$$

The assumption on $(\vec{a}, \vec{\alpha})$ and $(\vec{b}, \vec{\beta})$ gives an automorphism of $\left(\mathbf{k}^{\prime}, \Gamma^{\prime}\right)$ over $(\mathbf{k}, \Gamma)$ that takes $(\vec{a}, \vec{\alpha})$ to $(\vec{b}, \vec{\beta})$. This automorphism preserves the $\Sigma$-relations, so it extends to a field automorphism of $\mathbf{k}^{\prime}\left(\Gamma^{\prime}\right)$ over $\mathbf{k}(\Gamma)$, which extends further to a field automorphism of $\Omega^{\prime}$ over $\Omega$ by linear disjointness of $\mathbf{k}^{\prime}\left(\Gamma^{\prime}\right)$ and $\Omega$ over $\mathbf{k}(\Gamma)$. This yields the desired equality of types.

The implication $(3) \Rightarrow(2)$ of Theorem 1.2 is trivial, so in view of Lemma 4.2 and Proposition 4.5. this theorem will be established once we prove the part $(1) \Rightarrow(3)$, which is the next result.

Proposition 7.2. Suppose $(\Omega, \mathbf{k}, \Gamma) \models T$ and $(\mathbf{k}, \Gamma)$ is a Mann pair. Then every subset of $\mathbf{k}^{m} \times \Gamma^{n}$ that is definable in $(\Omega, \mathbf{k}, \Gamma)$ is a finite union of sets $X \times Y$ with $X \subseteq \mathbf{k}^{m}$ definable in the field $\mathbf{k}$ and $Y \subseteq \Gamma^{n}$ definable in the group $\Gamma$.

Proof. As in the proof of the previous lemma we take an $|\Omega|^{+}$-saturated elementary extension $\left(\Omega^{\prime}, \mathbf{k}^{\prime}, \Gamma^{\prime}\right)$ of $(\Omega, \mathbf{k}, \Gamma)$ and tuples $\vec{a}, \vec{b} \in\left(\mathbf{k}^{\prime}\right)^{m}$ and $\vec{\alpha}, \vec{\beta} \in\left(\Gamma^{\prime}\right)^{n}$ such that

$$
\operatorname{tp}_{\mathbf{k}^{\prime}}(\vec{a} \mid \mathbf{k})=\operatorname{tp}_{\mathbf{k}^{\prime}}(\vec{b} \mid \mathbf{k}) \text { and } \operatorname{tp}_{\Gamma^{\prime}}(\vec{\alpha} \mid \Gamma)=\operatorname{tp}_{\Gamma^{\prime}}(\vec{\beta} \mid \Gamma) .
$$

By Lemma 7.1 it is enough to show that then

$$
\operatorname{tp}_{\left(\mathbf{k}^{\prime}, \Gamma^{\prime}\right)}((\vec{a}, \vec{\alpha}) \mid(\mathbf{k}, \Gamma))=\operatorname{tp}_{\left(\mathbf{k}^{\prime}, \Gamma^{\prime}\right)}((\vec{b}, \vec{\beta}) \mid(\mathbf{k}, \Gamma)) .
$$

The assumption on $\vec{a}$ and $\vec{b}$ gives an automorphism $\sigma$ of $\mathbf{k}^{\prime}$ over $\mathbf{k}$ such that $\sigma(\vec{a})=\vec{b}$, and the assumption on $\vec{\alpha}$ and $\vec{\beta}$ gives an automorphism $\phi$ of $\Gamma^{\prime}$ over $\Gamma$ such that $\phi(\vec{\alpha})=\vec{\beta}$. It remains to show that this gives an automorphism $(\sigma, \phi)$ of the $\mathcal{L}_{\Sigma}^{\mathrm{f}, \mathrm{g}}$ structure $\left(\mathbf{k}^{\prime}, \Gamma^{\prime}\right)$. This in turn reduces to establishing the following: Let $N$ be a positive integer and $\vec{c}^{\prime} \in\left(\mathbf{k}^{\prime}\right)^{N}$ and $\vec{\gamma}^{\prime} \in\left(\Gamma^{\prime}\right)^{N}$. Then

$$
\left(\mathbf{k}^{\prime}, \Gamma^{\prime}\right) \models \Sigma_{N}\left(\vec{c}^{\prime}, \vec{\gamma}^{\prime}\right) \Longleftrightarrow\left(\mathbf{k}^{\prime}, \Gamma^{\prime}\right) \models \Sigma_{N}\left(\sigma\left(\vec{c}^{\prime}\right), \phi\left(\vec{\gamma}^{\prime}\right)\right) .
$$

We prove the forward implication. (The backward implication follows in the same way.) Assume $\left(\mathbf{k}^{\prime}, \Gamma^{\prime}\right) \models \Sigma_{N}\left(\vec{c}^{\prime}, \vec{\gamma}^{\prime}\right)$. We have $\Sigma_{N}(\mathbf{k}, \Gamma)=\bigcup_{i \in I} P_{i} \times Q_{i}$, where $I$ is 
finite, each $P_{i} \subseteq \mathbf{k}^{N}$ is definable in the field $\mathbf{k}$ and each $Q_{i} \subseteq \Gamma^{N}$ is definable in the group $\Gamma$. Then $\Sigma_{N}\left(\mathbf{k}^{\prime}, \Gamma^{\prime}\right)=\bigcup_{i \in I} P_{i}^{\prime} \times Q_{i}^{\prime}$, where $P_{i}^{\prime} \subseteq\left(\mathbf{k}^{\prime}\right)^{N}$ is defined in $\mathbf{k}^{\prime}$ by any formula with parameters from $\mathbf{k}$ that defines $P_{i}$ in the field $\mathbf{k}$, and $Q_{i}^{\prime} \subseteq\left(\Gamma^{\prime}\right)^{N}$ is defined in $\Gamma^{\prime}$ by any formula with parameters from $\Gamma$ that defines $Q_{i}$ in the group $\Gamma$. Take $i \in I$ such that $\vec{c}^{\prime} \in P_{i}^{\prime}$ and $\vec{\gamma}^{\prime} \in Q_{i}^{\prime}$. It is clear that then $\sigma\left(\vec{c}^{\prime}\right) \in P_{i}^{\prime}$ and $\phi\left(\vec{\gamma}^{\prime}\right) \in Q_{i}^{\prime}$, so $\left(\mathbf{k}^{\prime}, \Gamma^{\prime}\right) \models \Sigma_{N}\left(\sigma\left(\vec{c}^{\prime}\right), \phi\left(\vec{\gamma}^{\prime}\right)\right)$, as desired.

\section{The elementary theory of $\Omega$ With a Mann pair}

In this section we discuss various model-theoretic properties of models $(\Omega, \mathbf{k}, \Gamma)$ of $T$ where $(\mathbf{k}, \Gamma)$ is a Mann pair.

8.1. Elementary equivalence and smallness. We first prove that the theory of a model $(\Omega, \mathbf{k}, \Gamma)$ of $T$ is determined by the $\mathcal{L}_{\Sigma}^{\mathrm{f}, \mathrm{g}}$-theory of $(\mathbf{k}, \Gamma)$ whenever $\mathbf{k} \cup \Gamma$ is small in $\Omega$. For this we do not need $(\mathbf{k}, \Gamma)$ to be a Mann pair. (A definition and basic properties of small are in [5], Section 2.)

Lemma 8.1. Let $\left(\Omega_{1}, \mathbf{k}_{1}, \Gamma_{1}\right)$ and $\left(\Omega_{2}, \mathbf{k}_{2}, \Gamma_{2}\right)$ be models of $T$ such that $\mathbf{k}_{i} \cup \Gamma_{i}$ is small in $\Omega_{i}$ for $i=1,2$, and $\left(\mathbf{k}_{1}, \Gamma_{1}\right) \equiv\left(\mathbf{k}_{2}, \Gamma_{2}\right)$ as $\mathcal{L}_{\Sigma}^{\mathrm{f}, \mathrm{g}}$-structures. Then $\left(\Omega_{1}, \mathbf{k}_{1}, \Gamma_{1}\right) \equiv\left(\Omega_{2}, \mathbf{k}_{2}, \Gamma_{2}\right)$.

Proof. It is harmless to assume $\mathrm{CH}$ (the continuum hypothesis), so we can reduce to the case that $\left(\Omega_{i}, \mathbf{k}_{i}, \Gamma_{i}\right)$ is saturated of cardinality $\aleph_{1}$ for $i=1,2$. Then we have an $\mathcal{L}_{\Sigma}^{\mathrm{f}, \mathrm{g}}$-isomorphism

$$
\left(\iota_{\mathrm{f}}, \iota_{\mathrm{g}}\right):\left(\mathbf{k}_{1}, \Gamma_{1}\right) \rightarrow\left(\mathbf{k}_{2}, \Gamma_{2}\right) .
$$

This yields a ring isomorphism $\mathbf{k}_{1}\left[\Gamma_{1}\right] \rightarrow \mathbf{k}_{2}\left[\Gamma_{2}\right]$ extending both $\iota_{\mathrm{f}}$ and $\iota_{\mathrm{g}}$, and hence a field isomorphism

$$
\iota: \mathbf{k}_{1}\left(\Gamma_{1}\right)^{\mathrm{ac}} \rightarrow \mathbf{k}_{2}\left(\Gamma_{2}\right)^{\mathrm{ac}}
$$

By the smallness assumption the transcendence degree of $\Omega_{i}$ over $\mathbf{k}_{i}\left(\Gamma_{i}\right)^{\text {ac }}$ is $\aleph_{1}$ for $i=1,2$. Thus we can extend $\iota$ to a field isomorphism $\Omega_{1} \rightarrow \Omega_{2}$, which is then an $\mathcal{L}$-isomorphism.

Let $\mathbf{k}$ be a subfield of the algebraically closed field $\Omega$. A theorem of E. Artin says that if $1<[\Omega: \mathbf{k}]<\infty$, then $\mathbf{k}$ is real closed with $\Omega=\mathbf{k}(\sqrt{-1})$. As noted at the end of Section 2 in [5] it follows that by work of Keisler [8] the following are equivalent:

(1) $\mathbf{k}$ is small in $\Omega$;

(2) $[\Omega: \mathbf{k}]>2$;

(3) $[\Omega: \mathbf{k}]=\infty$.

We use this equivalence in the proof of the next lemma, which is analogous to Lemma 6.1 in [5]. (The proofs are not at all similar.)

Lemma 8.2. Assume $(\Omega, \mathbf{k}, \Gamma) \models T$, where $[\Omega: \mathbf{k}]>2$ and $(\mathbf{k}, \Gamma)$ is a Mann pair. Then $\mathbf{k} \cup \Gamma$ is small in $\Omega$.

Proof. We can assume that $\Gamma$ is infinite. Take a proper elementary extension $\left(\Omega^{*}, \mathbf{k}^{*}, \Gamma^{*}\right)$ of $(\Omega, \mathbf{k}, \Gamma)$. Using that $(\mathbf{k}, \Gamma)$ is a Mann pair, it follows easily that 
the subfields $\mathbf{k}^{*}$ and $\mathbf{k}\left(\Gamma^{*}\right)$ of $\Omega^{*}$ are linearly disjoint over $\mathbf{k}$. See the diagram below where all arrows are inclusions:

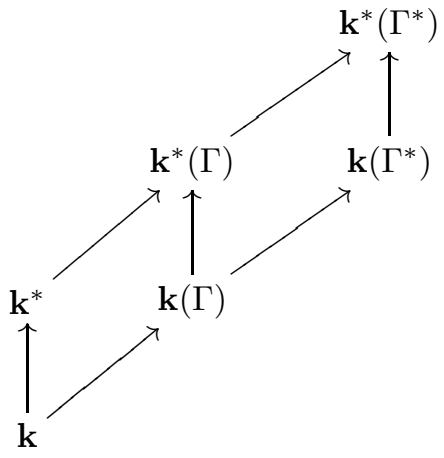

It follows that $\mathbf{k}^{*}(\Gamma)$ and $\mathbf{k}\left(\Gamma^{*}\right)$ are linearly disjoint over $\mathbf{k}(\Gamma)$. Among the subsets of $\Gamma^{*}$ that are multiplicatively independent over $\Gamma$, take one, say $B$, that is maximal. It follows from Lemma 5.12 in [5] that $B$ is algebraically independent over $\mathbf{k}(\Gamma)$. Hence $B$ is algebraically independent over $\mathbf{k}^{*}(\Gamma)$. The maximality property of $B$ guarantees that $B \neq \emptyset$ and for every $\gamma \in \Gamma^{*}$ there is a positive integer $d$ with $\gamma^{d} \in$ $\mathbf{k}^{*}(\Gamma)(B)$. Since $\mathbf{k}^{*}(\Gamma)(B)$ is a nontrivial purely transcendental extension of $\mathbf{k}^{*}(\Gamma)$ it follows that $\mathbf{k}^{*}\left(\Gamma^{*}, \sqrt{-1}\right)$ is not algebraically closed, and so $\mathbf{k}^{*}\left(\Gamma^{*}, \sqrt{-1}\right) \neq \Omega^{*}$. Hence by the remark preceding this lemma, $\mathbf{k}^{*} \cup \Gamma^{*}$ is small in $\Omega^{*}$, and thus $\mathbf{k} \cup \Gamma$ is small in $\Omega$; see Section 2 in $[5$.

\subsection{Stability.}

Proposition 8.3. Let $(\Omega, \mathbf{k}, \Gamma) \models T$ be such that $\mathbf{k}$ is algebraically closed and $(\mathbf{k}, \Gamma)$ is a Mann pair. Then $(\Omega, \mathbf{k}, \Gamma)$ is stable, and if $\Gamma$ is divisible, then $(\Omega, \mathbf{k}, \Gamma)$ is $\omega$-stable.

Proof. Take an infinite cardinal $\kappa$ such that the abelian group $\Gamma$ is $\kappa$-stable. We show that then $(\Omega, \mathbf{k}, \Gamma)$ is $\kappa$-stable. We can assume $\mathbf{k} \neq \Omega$, and that $|\Omega|=\kappa$. Take a $\kappa^{+}$-saturated elementary extension $\left(\Omega^{\prime}, \mathbf{k}^{\prime}, \Gamma^{\prime}\right)$ of $(\Omega, \mathbf{k}, \Gamma)$.

By the proofs of Lemma 7.1 and Proposition 7.2 , the type of an element of $\mathbf{k}^{\prime}$ over $\Omega$ in $\left(\Omega^{\prime}, \mathbf{k}^{\prime}, \Gamma^{\prime}\right)$ is determined by its type over $\mathbf{k}$ in the field $\mathbf{k}^{\prime}$. Likewise, the type of an element of $\Gamma^{\prime}$ over $\Omega$ in $\left(\Omega^{\prime}, \mathbf{k}^{\prime}, \Gamma^{\prime}\right)$ is determined by its type over $\Gamma$ in the group $\Gamma^{\prime}$.

Now let $t \in \Omega\left(\mathbf{k}^{\prime} \cup \Gamma^{\prime}\right)^{\text {ac }}$, say $t \in \Omega(\vec{a}, \vec{\gamma})^{\text {ac }}$ with $\vec{a} \in\left(\mathbf{k}^{\prime}\right)^{m}$ and $\vec{\gamma} \in\left(\Gamma^{\prime}\right)^{n}$. Then $\operatorname{tp}_{\left(\Omega^{\prime}, \mathbf{k}^{\prime}, \Gamma^{\prime}\right)}(t \mid \Omega)$ is determined by $\operatorname{tp}_{\mathbf{k}^{\prime}}(\vec{a} \mid \mathbf{k}), \operatorname{tp}_{\Gamma^{\prime}}(\vec{\gamma} \mid \Gamma)$ and the specification of a polynomial $P(X, Y, T) \in \Omega[X, Y, T]$ where $X=\left(X_{1}, \ldots, X_{m}\right), Y=\left(Y_{1}, \ldots, Y_{n}\right)$ and $T$ is a single indeterminate such that $P(\vec{a}, \vec{\gamma}, T) \in \Omega(\vec{a}, \vec{\gamma})[T]$ is irreducible and $P(\vec{a}, \vec{\gamma}, t)=0$.

Finally, by the last argument of the proof of Lemma 8.1, all elements of $\Omega^{\prime}$ outside $\Omega\left(\mathbf{k}^{\prime} \cup \Gamma^{\prime}\right)^{\text {ac }}$ realize the same type in $\left(\Omega^{\prime}, \mathbf{k}^{\prime}, \Gamma^{\prime}\right)$ over $\Omega$.

Hence we have at most $\kappa$ many different 1 -types in $\left(\Omega^{\prime}, \mathbf{k}^{\prime}, \Gamma^{\prime}\right)$ over $\Omega$.

Remark. One can show that if $(\Omega, \mathbf{k}, \Gamma)$ is a model of $T$ and $(\mathbf{k}, \Gamma)$ is a Mann pair, then the subsets $\mathbf{k}$ and $\Gamma$ of $\Omega$ are definable in the structure $(\Omega, \mathbf{k} \cup \Gamma)$. Using this fact, Proposition 8.3 also follows from Fact 2.1 and Theorem 4.8 in [1]. 
8.3. Axiomatizing $(\Omega, \mathbf{k}, \Gamma)$. Our goal here is to show that if $(\Omega, \mathbf{k}, \Gamma)$ is a model of $T$ and $(\mathbf{k}, \Gamma)$ is a Mann pair with $\mathbf{k}$ small in $\Omega$, then the elementary theory of $(\Omega, \mathbf{k}, \Gamma)$ is completely determined by the elementary theories of the field $\mathbf{k}$ and of the group $\Gamma$. We achieve this after adding names for enough elements of $\mathbf{k}$ and $\Gamma$ to witness that $(\mathbf{k}, \Gamma)$ is a Mann pair.

Fix a model $\left(\Omega_{0}, \mathbf{k}_{0}, \Gamma_{0}\right)$ of $T$ such that $\left(\mathbf{k}_{0}, \Gamma_{0}\right)$ is a Mann pair. For each $n \geq 2$, fix a finite subset $\Gamma_{0}(n)$ of $\Gamma_{0}^{n}$ such that

$$
\Sigma_{n}^{\mathrm{nd}}\left(\mathbf{k}_{0}, \Gamma_{0}\right)=\bigcup_{\vec{\gamma} \in \Gamma_{0}(n)} \Sigma_{n}^{\mathrm{nd}}\left(\mathbf{k}_{0}, \Gamma_{0} ; \vec{\gamma}\right) \times \Gamma_{0} \vec{\gamma},
$$

and for each $\vec{\gamma} \in \Gamma_{0}(n)$, fix a basis $B(\vec{\gamma}) \subseteq \mathbf{k}_{0}^{n}$ of the $\mathbf{k}_{0}$-linear subspace $\Sigma_{n}\left(\mathbf{k}_{0}, \Gamma_{0} ; \vec{\gamma}\right)$ of $\mathbf{k}_{0}^{n}$. Let $\mathcal{L}\left(\mathbf{k}_{0}, \Gamma_{0}\right)$ be the language $\mathcal{L}$ augmented by names for the elements of $\mathbf{k}_{0} \cup \Gamma_{0}$.

In the following definition and subsequent remarks, $(\Omega, \mathbf{k}, \Gamma)$ ranges over models of $T$ that contain $\left(\Omega_{0}, \mathbf{k}_{0}, \Gamma_{0}\right)$ as a substructure. We construe such $(\Omega, \mathbf{k}, \Gamma)$ as an $\mathcal{L}\left(\mathbf{k}_{0}, \Gamma_{0}\right)$-structure in the obvious way, and we say that $(\Omega, \mathbf{k}, \Gamma)$ satisfies the Mann-axioms of $\left(\Omega_{0}, \mathbf{k}_{0}, \Gamma_{0}\right)$ if for each $n \geq 2$ :

$$
\Sigma_{n}^{\mathrm{nd}}(\mathbf{k}, \Gamma)=\bigcup_{\vec{\gamma} \in \Gamma_{0}(n)} \Sigma_{n}^{\mathrm{nd}}(\mathbf{k}, \Gamma ; \vec{\gamma}) \times \Gamma \vec{\gamma} ;
$$

(2) for each $\vec{\gamma} \in \Gamma_{0}(n)$, the $\mathbf{k}$-linear subspace $\Sigma_{n}(\mathbf{k}, \Gamma ; \vec{\gamma})$ of $\mathbf{k}^{n}$ is generated by $B(\vec{\gamma})$.

Remarks. The reason for this terminology is that we have a set $\operatorname{Mann}\left(\mathbf{k}_{0}, \Gamma_{0}\right)$ of sentences in the language $\mathcal{L}\left(\mathbf{k}_{0}, \Gamma_{0}\right)$ such that for all $(\Omega, \mathbf{k}, \Gamma)$ as above,

$$
\begin{aligned}
(\Omega, \mathbf{k}, \Gamma) & \models \operatorname{Mann}\left(\mathbf{k}_{0}, \Gamma_{0}\right) \\
& \Longleftrightarrow
\end{aligned}
$$

$(\Omega, \mathbf{k}, \Gamma)$ satisfies the Mann axioms of $\left(\Omega_{0}, \mathbf{k}_{0}, \Gamma_{0}\right)$.

In particular, if $(\Omega, \mathbf{k}, \Gamma)$ is an elementary extension of $\left(\Omega_{0}, \mathbf{k}_{0}, \Gamma_{0}\right)$, then $(\Omega, \mathbf{k}, \Gamma)$ satisfies the Mann axioms of $\left(\Omega_{0}, \mathbf{k}_{0}, \Gamma_{0}\right)$.

Let $\mathcal{L}^{\mathrm{f}}:=\{0,1,-,+, \cdot\}$ be the one-sorted language whose symbols are those of $\mathcal{L}_{\Sigma}^{\mathrm{f}, \mathrm{g}}$ involving only sort $\mathrm{f}$, and let $\mathcal{L}^{\mathrm{f}}\left(\mathbf{k}_{0}\right)$ be $\mathcal{L}^{\mathrm{f}}$ augmented by names for the elements of $\mathbf{k}_{0}$. Let $\mathcal{L}^{\mathrm{g}}:=\left\{1,{ }^{-1}, \cdot\right\}$ be the one-sorted language whose symbols are those of $\mathcal{L}_{\Sigma}^{\mathrm{f}, \mathrm{g}}$ involving only sort g, and let $\mathcal{L}^{\mathrm{g}}\left(\Gamma_{0}\right)$ be $\mathcal{L}^{\mathrm{g}}$ augmented by names for the elements of $\Gamma_{0}$. Let $n \geq 1$, let $x=\left(x_{1}, \ldots, x_{n}\right)$ be a tuple of distinct f-variables, and let $y=\left(y_{1}, \ldots, y_{n}\right)$ be a tuple of distinct g-variables. A careful look at the proof of Lemma 4.2 for $\vec{r}=(1, \ldots, 1)$ yields the following: there are quantifier-free $\mathcal{L}^{\mathrm{f}}\left(\mathbf{k}_{0}\right)$-formulas $\phi_{1}(x), \ldots, \phi_{m}(x)$ and quantifier-free $\mathcal{L}^{\mathrm{g}}\left(\Gamma_{0}\right)$-formulas $\psi_{1}(y), \ldots, \psi_{m}(y)$ such that for all $(\Omega, \mathbf{k}, \Gamma) \models \operatorname{Mann}\left(\mathbf{k}_{0}, \Gamma_{0}\right)$,

$$
\Sigma_{n}\left(\mathbf{k}^{n}, \Gamma^{n}\right)=\bigcup_{i=1}^{m} \phi_{i}\left(\mathbf{k}^{n}\right) \times \psi_{i}\left(\Gamma^{n}\right) .
$$

This uniformity is crucial in the proof of the next result.

Theorem 8.4. Let $\left(\Omega_{1}, \mathbf{k}_{1}, \Gamma_{1}\right)$ and $\left(\Omega_{2}, \mathbf{k}_{2}, \Gamma_{2}\right)$ be models of $T$ such that

(1) $\left[\Omega_{1}: \mathbf{k}_{1}\right]>2$ and $\left[\Omega_{2}: \mathbf{k}_{2}\right]>2$;

(2) $\left(\Omega_{0}, \mathbf{k}_{0}, \Gamma_{0}\right) \subseteq\left(\Omega_{1}, \mathbf{k}_{1}, \Gamma_{1}\right)$ and $\left(\Omega_{0}, \mathbf{k}_{0}, \Gamma_{0}\right) \subseteq\left(\Omega_{2}, \mathbf{k}_{2}, \Gamma_{2}\right)$;

(3) $\left(\Omega_{1}, \mathbf{k}_{1}, \Gamma_{1}\right)$ and $\left(\Omega_{2}, \mathbf{k}_{2}, \Gamma_{2}\right)$ satisfy the Mann-axioms of $\left(\Omega_{0}, \mathbf{k}_{0}, \Gamma_{0}\right)$.

Then: $\left(\Omega_{1}, \mathbf{k}_{1}, \Gamma_{1}\right) \equiv_{\mathbf{k}_{0} \cup \Gamma_{0}}\left(\Omega_{2}, \mathbf{k}_{2}, \Gamma_{2}\right) \Longleftrightarrow \mathbf{k}_{1} \equiv_{\mathbf{k}_{0}} \mathbf{k}_{2}$ and $\Gamma_{1} \equiv_{\Gamma_{0}} \Gamma_{2}$. 
Proof. The forward direction is clear. For the converse, assume $\mathbf{k}_{1} \equiv_{\mathbf{k}_{0}} \mathbf{k}_{2}$ and $\Gamma_{1} \equiv_{\Gamma_{0}} \Gamma_{2}$. Without loss we can also assume that $\left(\Omega_{1}, \mathbf{k}_{1}, \Gamma_{1}\right)$ and $\left(\Omega_{2}, \mathbf{k}_{2}, \Gamma_{2}\right)$ are $\kappa$-saturated, where $\kappa>\left|\mathbf{k}_{0}\right|+\left|\Gamma_{0}\right|$.

We construct a back-and-forth system between the $\mathcal{L}_{\Sigma}^{\mathrm{f}, \mathrm{g}}$-structures $\left(\mathbf{k}_{1}, \Gamma_{1}\right)$ and $\left(\mathbf{k}_{2}, \Gamma_{2}\right)$. Let $\mathcal{S}_{1}$ be the set of substructures $\left(\mathbf{k}_{1}^{\prime}, \Gamma_{1}^{\prime}\right)$ of $\left(\mathbf{k}_{1}, \Gamma_{1}\right)$ that extend $\left(\mathbf{k}_{0}, \Gamma_{0}\right)$ and have cardinality $<\kappa$ (so $\mathbf{k}_{1}^{\prime}$ is a subring, not necessarily a subfield, of $\mathbf{k}_{1}$, and $\Gamma_{1}^{\prime}$ is a subgroup of $\left.\Gamma_{1}\right)$. Define $\mathcal{S}_{2}$ likewise, with $\left(\mathbf{k}_{2}, \Gamma_{2}\right)$ in place of $\left(\mathbf{k}_{1}, \Gamma_{1}\right)$, and let $\mathcal{I}$ be the set of isomorphisms

$$
\iota=\left(\iota_{\mathrm{f}}, \iota_{\mathrm{g}}\right):\left(\mathbf{k}_{1}^{\prime}, \Gamma_{1}^{\prime}\right) \rightarrow\left(\mathbf{k}_{2}^{\prime}, \Gamma_{2}^{\prime}\right),
$$

where $\left(\mathbf{k}_{i}^{\prime}, \Gamma_{i}^{\prime}\right) \in \mathcal{S}_{i}$ for $i=1,2$, such that $\iota$ is the identity on $\left(\mathbf{k}_{0}, \Gamma_{0}\right), \iota_{\mathrm{f}}: \mathbf{k}_{1}^{\prime} \rightarrow \mathbf{k}_{2}^{\prime}$ is a partial elementary map from $\mathbf{k}_{1}$ to $\mathbf{k}_{2}$, and $\iota_{\mathrm{g}}: \Gamma_{1}^{\prime} \rightarrow \Gamma_{2}^{\prime}$ is a partial elementary map from $\Gamma_{1}$ to $\Gamma_{2}$. It is clear that the identity map of $\left(\mathbf{k}_{0}, \Gamma_{0}\right)$ belongs to $\mathcal{I}$.

Next we show that $\mathcal{I}$ is a back-and-forth system. By symmetry it is enough to show that we can go forth. Let

$$
\iota=\left(\iota_{\mathrm{f}}, \iota_{\mathrm{g}}\right):\left(\mathbf{k}_{1}^{\prime}, \Gamma_{1}^{\prime}\right) \rightarrow\left(\mathbf{k}_{2}^{\prime}, \Gamma_{2}^{\prime}\right)
$$

be in $\mathcal{I}$. Replacing $\mathbf{k}_{1}^{\prime}$ and $\mathbf{k}_{2}^{\prime}$ by their fraction fields inside $\mathbf{k}_{1}$ and $\mathbf{k}_{2}$ and extending $\iota_{\mathrm{f}}$ accordingly, without changing $\iota_{\mathrm{g}}$, we arrange that $\mathbf{k}_{1}^{\prime}$ and $\mathbf{k}_{2}^{\prime}$ are subfields of $\mathbf{k}_{1}$ and $\mathbf{k}_{2}$.

Let $a \in \mathbf{k}_{1} \backslash \mathbf{k}_{1}^{\prime}$ be given. Using saturation we can take $b \in \mathbf{k}_{2} \backslash \mathbf{k}_{2}^{\prime}$ and a field isomorphism $j: \mathbf{k}_{1}^{\prime}(a) \rightarrow \mathbf{k}_{2}^{\prime}(b)$ that extends $\iota_{\mathrm{f}}$, sends $a$ to $b$, and is a partial elementary map from $\mathbf{k}_{1}$ to $\mathbf{k}_{2}$. It is obvious that $\left(\mathbf{k}_{1}^{\prime}(a), \Gamma_{1}^{\prime}\right) \in \mathcal{S}_{1}$ and $\left(\mathbf{k}_{2}^{\prime}(b), \Gamma_{2}^{\prime}\right) \in$ $\mathcal{S}_{2}$, and it follows easily from the remark just before the lemma that $\left(j, \iota_{\mathrm{g}}\right) \in \mathcal{I}$.

Now let $\alpha \in \Gamma_{1} \backslash \Gamma_{1}^{\prime}$. By saturation we can find $\beta \in \Gamma_{2} \backslash \Gamma_{2}^{\prime}$ and a group isomorphism $h: \alpha^{\mathbb{Z}} \Gamma_{1}^{\prime} \rightarrow \beta^{\mathbb{Z}} \Gamma_{2}^{\prime}$ from $\Gamma_{1}$ to $\Gamma_{2}$ that extends $\iota_{\mathrm{g}}$, sends $\alpha$ to $\beta$, and is a partial elementary map from $\Gamma_{1}$ to $\Gamma_{2}$. It is obvious that $\left(\mathbf{k}_{1}^{\prime}, \alpha^{\mathbb{Z}} \Gamma_{1}^{\prime}\right) \in \mathcal{S}_{1}$ and $\left(\mathbf{k}_{2}^{\prime}, \beta^{\mathbb{Z}} \Gamma_{2}^{\prime}\right) \in \mathcal{S}_{2}$. It follows easily from the remark just before the lemma that $\left(\iota_{\mathrm{f}}, h\right):\left(\mathbf{k}_{1}^{\prime}, \alpha^{\mathbb{Z}} \Gamma_{1}^{\prime}\right) \rightarrow\left(\mathbf{k}_{2}^{\prime}, \beta^{\mathbb{Z}} \Gamma_{2}^{\prime}\right)$ belongs to $\mathcal{I}$.

We have now shown that $\mathcal{I}$ is a nonempty back-and-forth system. It follows that $\left(\mathbf{k}_{1}, \Gamma_{1}\right) \equiv_{\left(\mathbf{k}_{0}, \Gamma_{0}\right)}\left(\mathbf{k}_{2}, \Gamma_{2}\right)$. From (1), (3), and Lemma 8.2 we obtain that $\mathbf{k}_{i} \cup \Gamma_{i}$ is small in $\Omega_{i}$ for $i=1,2$. Then a proof such as that of Lemma 8.1 yields

$$
\left(\Omega_{1}, \mathbf{k}_{1}, \Gamma_{1}\right) \equiv_{\mathbf{k}_{0} \cup \Gamma_{0}}\left(\Omega_{2}, \mathbf{k}_{2}, \Gamma_{2}\right) \Longleftrightarrow \mathbf{k}_{1} \equiv_{\mathbf{k}_{0}} \mathbf{k}_{2} \text { and } \Gamma_{1} \equiv_{\Gamma_{0}} \Gamma_{2},
$$

as desired.

For algebraically closed $\mathbf{k}_{i}$, this result takes the following form:

Corollary 8.5. Let $\left(\Omega_{1}, \mathbf{k}_{1}, \Gamma_{1}\right)$ and $\left(\Omega_{2}, \mathbf{k}_{2}, \Gamma_{2}\right)$ be models of $T$ such that

(1) $\mathbf{k}_{1}$ and $\mathbf{k}_{2}$ are algebraically closed, $\mathbf{k}_{1} \neq \Omega_{1}, \mathbf{k}_{2} \neq \Omega_{2}$;

(2) $\left(\Omega_{0}, \mathbf{k}_{0}, \Gamma_{0}\right) \subseteq\left(\Omega_{1}, \mathbf{k}_{1}, \Gamma_{1}\right)$ and $\left(\Omega_{0}, \mathbf{k}_{0}, \Gamma_{0}\right) \subseteq\left(\Omega_{2}, \mathbf{k}_{2}, \Gamma_{2}\right)$;

(3) $\left(\Omega_{1}, \mathbf{k}_{1}, \Gamma_{1}\right)$ and $\left(\Omega_{2}, \mathbf{k}_{2}, \Gamma_{2}\right)$ satisfy the Mann axioms of $\left(\Omega_{0}, \mathbf{k}_{0}, \Gamma_{0}\right)$.

Then: $\left(\Omega_{1}, \mathbf{k}_{1}, \Gamma_{1}\right) \equiv_{\mathbf{k}_{0} \cup \Gamma_{0}}\left(\Omega_{2}, \mathbf{k}_{2}, \Gamma_{2}\right) \Longleftrightarrow \Gamma_{1} \equiv_{\Gamma_{0}} \Gamma_{2}$.

\section{ACKNOWLEDGEMENT}

The referee has pointed out that Theorem 1.1 also follows from Hrushovski and Pillay [7. At the end of Section 6 we indicate how. The referee also mentioned that Sections 7 and 8 are related to earlier work by Casanovas and Ziegler 4 and by Pillay [13]. 


\section{REFERENCES}

1. B. Baizhanov, J.T. Baldwin, Local Homogeneity, Journal of Symbolic Logic, 69, (2004), pp. 1243-1260. MR2135665 (2006e:03043)

2. J. Boxall, Sous-variétés algébriques de variétés semi-abéliennes sur un corps fini, Number Theory (Paris 1992-1993), 69-80, London Math. Soc. Lecture Note Ser., 215, Cambridge Univ. Press, Cambridge, 1995. MR.1345173 (96k:14037)

3. W.D. Brownawell, D.W. Masser, Vanishing sums in function fields, Math. Proc. Camb. Phil. Soc., 100, (1986), pp. 427-434. MR857720 (87k:11080)

4. E. Casanovas, M. Ziegler, Stable theories with a new predicate, Journal of Symbolic Logic 66 (2001), no.3, 1127-1140. MR1856732 (2002k:03050)

5. L. VAN DEN Dries, A. Günaydin, The fields of real and complex numbers with a small multiplicative group, Proceedings of London Mathematical Society (3), 93, (2006), pp. 43-81. MR2235481 (2007i:03039)

6. J.H. Evertse, On sums of $S$-units and linear recurrences, Compositio Math. 53 (1984), 225244. MR766298 (86c:11045)

7. E. Hrushovski, A. Pillay, Effective bounds for the number of transcendental points on subvarieties of semi-abelian varieties, American Journal of Mathematics, 122 (2000), 439450. MR1759883 (2001d:11078)

8. H.J. KEISLER, Complete theories of algebraically closed fields with distinguished subfields, Michigan Math. J. 11 (1964), 71-81. MR0179080 (31:3331)

9. S. Lang, Algebra, Addison-Wesley Publishing Co. Inc., Reading, Mass., 1997. xvi+912 pp. MR 783636 (86j:00003)

10. S. LANG, Introduction to algebraic and abelian functions (Second edition), Graduate Texts in Mathematics, 89, Springer-Verlag, New York and Berlin, 1982. ix+169 pp. MR681120 (84m:14032)

11. M. Laurent, Équations diophantiennes exponentielles, Invent. Math. 78 (1984), 299-327. MR.767195 (86j:11062)

12. H. ManN, On Linear Relations Between Roots of Unity, Mathematika 12 (1965), 107-117. MR0191892 (33:119)

13. A. PILlAY, The model-theoretic content of Lang's conjecture, in BousCAREN ET AL. EDS., Model Theory and Algebraic Geometry, Springer Lecture Notes in Math., 1696, 101-106 (1998). MR.1678531 (2000c:11203)

14. A.J. van Der Poorten, H.P. Schlickewei, Additive relations in fields, J. Australian Math. Soc. 51 (1991), 154-170. MR.1119694 (93d:11036)

15. T. SCANLON, J.F. VOLOCH, Difference algebraic subgroups of commutative algebraic groups over finite fields, Manuscripta Math. 99 (1999), 329-339. MR1702597 (2000i:12009)

Department of Mathematics, University of Illinois, 1409 W. Green Street, Urbana, ILLINOIS 61801

E-mail address: vddries@math.uiuc.edu

Fields Institute, 222 College Street, Second Floor, Toronto, Ontario, Canada M5T 3J1

E-mail address: agunaydi@fields.utoronto.ca 